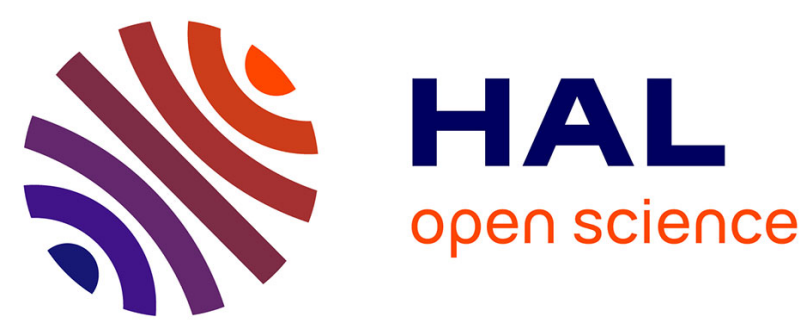

\title{
Vulnérabilité métabolique et potentialités des milieux insulaires. Le cas de l'île de Ndzuwani (Anjouan), archipel des Comores
}

\author{
Jean-Baptiste Bahers, Jeanne Perez, Mathieu Durand
}

\section{To cite this version:}

Jean-Baptiste Bahers, Jeanne Perez, Mathieu Durand. Vulnérabilité métabolique et potentialités des milieux insulaires. Le cas de l'île de Ndzuwani (Anjouan), archipel des Comores. Flux - Cahiers scientifiques internationaux Réseaux et territoires, 2019, №128-146 (2), pp.128. 10.3917/flux1.116.0128 . halshs-02462461

\section{HAL Id: halshs-02462461 \\ https://shs.hal.science/halshs-02462461}

Submitted on 4 Feb 2020

HAL is a multi-disciplinary open access archive for the deposit and dissemination of scientific research documents, whether they are published or not. The documents may come from teaching and research institutions in France or abroad, or from public or private research centers.
L'archive ouverte pluridisciplinaire HAL, est destinée au dépôt et à la diffusion de documents scientifiques de niveau recherche, publiés ou non, émanant des établissements d'enseignement et de recherche français ou étrangers, des laboratoires publics ou privés. 


\section{Vulnérabilité métabolique et potentialités des milieux insulaires. Le cas de l'île de Ndzuwani (Anjouan), archipel des Comores}

\section{Introduction}

1Les milieux insulaires présentent un intérêt particulier pour les études du métabolisme territorial (Barles, 2017), car leur relatif isolement et les contraintes physiques imposent potentiellement une certaine limitation des flux de matière et d'énergie, ou du moins une tentative de rationalisation. Certains territoires insulaires connaissent des contraintes supplémentaires concernant leur développement économique. On les nomme les Petits États Insulaires en Développement (PEID), qui ont en commun de concentrer des vulnérabilités sociales, territoriales et environnementales (Bahers, 2011 ; Taglioni, 2008). L'Union des Comores, composée de plusieurs îles, est un de ces PEID. Nous avons ici étudié le métabolisme de l'île de Ndzuwani (Anjouan dans le langage francophone).

2De nombreuses activités économiques sont informelles et non recensées à Ndzuwani, notamment la gestion des déchets, la construction et l'agriculture. Ce contexte d'informalité très prégnant, bien connu en rudologie (Millington, Lawhon, 2018), conduit à relever un défi méthodologique dans le but de mener une étude de métabolisme avec l'outil d'Analyse des Flux de Matières (AFM [1][1]Traduit de MFA : Material Flow Analysis.), ce qui est très peu abordé par ce registre d'études. En effet, l'AFM nécessite, dans la plupart des cas, un croisement de nombreuses bases de données (Bahers, Barles, Durand, 2019). Comment établir une méthode de quantification des flux de matière et d'énergie lorsqu'une majorité des activités économiques est exercée par des acteurs informels ? Ainsi, dans le cas de cette recherche, nous avons croisé des données issues des importations et exportations par le port de marchandises avec les discours et les informations recueillies auprès des acteurs du territoire. Cela s'est fait à travers une enquête de terrain approfondie accompagnée d'un comité de parties prenantes (institutions municipales et gouvernementales, ONG locales et internationales, entreprises privées et associatives). Par le biais de la proposition du concept de vulnérabilité métabolique, nous avons enfin investigué les conséquences sociales et environnementales du métabolisme insulaire. L'objectif de l'article est de montrer en quoi la connaissance de la circulation des flux de matière et d'énergie qui traverse un territoire insulaire permet de mieux saisir les vulnérabilités qui en résultent.

3LLa première partie s'attache à comprendre les spécificités de la gestion des déchets et ressources insulaires et à mobiliser les trois écologies scientifiques qui s'approprient la notion de métabolisme insulaire. Cela nous conduit à construire un cadre d'analyse des vulnérabilités 
métaboliques. Dans une deuxième partie, est présenté le cas d'étude. Puis, l'adaptation de l'AFM aux contraintes de l'île de Ndzuwani est discutée sous l'angle des données et du déroulé de l'enquête. Enfin, la dernière partie présente la circulation des flux de matières et d'énergie, ainsi que leurs trajectoires spatiales. Cela nous conduit à exposer trois situations de vulnérabilité métabolique, mais également à montrer les forces du métabolisme de l'île de Ndzuwani.

\section{Métabolismes insulaires et vulnérabilité dans les PEID}

4Avant d'analyser les vulnérabilités métaboliques de Ndzuwani, il est important de situer les champs théoriques qui discutent du métabolisme insulaire. Ensuite, nous aborderons les spécificités de la gestion des déchets et des ressources dans les PEID afin d'en saisir les enjeux opérationnels, avant de les croiser avec l'analyse des vulnérabilités environnementales selon un regard interdisciplinaire.

\section{Le métabolisme insulaire : un archipel scientifique}

5Le concept de métabolisme insulaire est discuté par les trois écologies scientifiques (Newell, Cousins, 2015 ; Wachsmuth, 2012), que sont l'écologie industrielle, l'écologie sociale et l'écologie politique. Cette section permet de présenter les distinctions dans leur appropriation conceptuelle.

6Depuis le travail pionnier de Chertow (Deschenes, Chertow, 2004 ; Eckelman, Chertow, 2009), les chercheurs en écologie industrielle se sont peu intéressés aux territoires insulaires, malgré les perspectives passionnantes du fait de la fermeture territoriale des flux. Par ailleurs, ces travaux sont pour l'essentiel prescriptifs et visent la mise en œuvre de synergies industrielles, entendues comme l'échange de co-produits ou de déchets entre acteurs économiques. Ils s'interrogent peu sur les facteurs socio-économiques ou sur les profils métaboliques.

7Du côté de l'écologie sociale, on peut noter des travaux récents de l'Institut du même nom sur l'Islande, Trinidad ou Samothraki en Grèce (Fetzel et alii, 2018 ; Krausmann, Richter, Eisenmenger, 2014 ; Petridis, Fischer-Kowalski, 2016 ; Singh et alii, 2001). Y sont développées des méthodes d'analyse du métabolisme insulaire, dans lesquelles est exploité un couplage de l'approche par l'indicateur de consommation intérieure de matières (Domestic Material Consumption - DMC) et d'indicateurs économiques sur le long terme, afin d'analyser la soutenabilité locale. Il en résulte que ces territoires exploitent des ressources naturelles très dépendantes des marchés mondiaux. De plus, ces types d'exploitation (mines ou énergies fossiles) conduisent à une production de déchets démesurée par rapport au nombre d'habitants. La perspective de productivité de la ressource est donc primordiale dans ce contexte. Ces territoires exportent la majeure partie de leur production et importent la quasi-totalité de leurs besoins alimentaires, énergétiques ou en matériaux selon le cas. 
8De même, deux autres travaux récents sont parus dans ce champ de recherche pour évaluer les politiques locales en matière de consommation de combustibles fossiles et de production agricole aux îles Galápagos (Cecchin, 2016) et du point de vue du métabolisme énergétique aux îles Baléares (Ginard-Bosch, Ramos-Martín, 2016). Ils conduisent à des recommandations vers des îles low-carbon et plus résilientes. Martinico-Perez et alii (2018) ont mené une étude du métabolisme socio-économique de l'archipel des Philippines qui met en parallèle les consommations de ressources et l'évolution du produit intérieur brut afin de comprendre l'évolution des pressions environnementales. Pour cette économie émergente, ils montrent un relatif découplage entre intensité matérielle et croissance économique, notamment du fait de la servicisation de l'économie.

9Les chercheurs s'inscrivant dans le courant de l'écologie politique ont également utilisé les territoires insulaires pour développer des corpus empiriques, que ce soit pour critiquer l'impact environnemental du tourisme (Gössling, 2003), la gestion de l'eau (Cole, 2012), la gestion forestière (Thompson, 2018) ou l'extraction matérielle (Allen, 2017). Au-delà de l'étude les déterminants de la vulnérabilité aux changements climatiques des territoires insulaires (Pelling, Uitto, 2001), la notion de « island energy metabolism » [2][2]Que nous traduisons par «métabolisme énergétique insulaire ». est avancée par (Harrison, Popke, 2018, p. 165) comme moyen de conceptualiser les relations entre les propriétés biophysiques de différentes sources d'énergie et les caractéristiques distinctives territoriales, infrastructurelles et géopolitiques des îles.

10Ainsi, comme pour les approches urbaines du métabolisme (Barles, 2010 ; Breetz, 2017 ; Newell, Cousins, 2015 ; Wachsmuth, 2012), les courants scientifiques dits d'écologie industrielle, de l'écologie sociale et de l'écologie politique urbaine sont pour l'instant très cloisonnés concernant les cas d'études insulaires. Les objectifs avancés dans cet article sont justement de croiser les approches vers une analyse socio-matérielle des métabolismes insulaires.

\section{Les défis de la gestion des déchets et des ressources insulaires}

$\underline{11}$ Les enjeux de production et de traitement des déchets sont particulièrement exacerbés dans un contexte insulaire, notamment dans les PEID. Certains archipels sont aussi terriblement célèbres à cause de leurs déchets, comme Samoa dont la décharge à ciel ouvert a largement atteint sa limite de capacité, Nauru qui croule sous les déchets miniers et dont les eaux sont polluées par le phosphate, Corfou qui accueille les touristes avec une montagne de 4000 tonnes de déchets. D'autres sont fonctionnellement dédiées aux déchets, comme les îlespoubelles de Thilafushi en Indonésie ou Semakau à Singapour, suivant le syndrome du dévastateur court métrage $L$ 'Île aux fleurs [3][3]Ilhadas Flores, de Jorge Furtado, Brésil, 1989. de Jorge Frutado. Il y a aussi de nombreuses îles dont les plages sont jonchées de déchets rapportés par les courants marins comme celle d'Henderson [4][4]L'île Henderson est une des quatre îles Pitcairn, territoire... (Bond, Lavers, 2017).

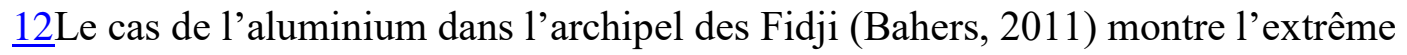
déséquilibre de la filière : la majorité de ce matériau est importée, que ce soit sous une forme semi-finie ou manufacturée, et seulement $5 \%$ du gisement des déchets est collecté pour être recyclé... à l'export. Le reste est stocké dans des décharges contrôlées ou sauvages. Par 
ailleurs, cette situation est exacerbée par les déséquilibres commerciaux entre l'archipel et ses partenaires. Par exemple, certains chalutiers japonais rapportent, en échange de l'acceptation des autorités fidjiennes à sur-pêcher dans leurs mers, des voitures à des prix abordables de deuxième ou troisième main... qui finissent très vite dans des décharges puisque les infrastructures de traitement manquent sur l'île.

13Cette problématique rejoint la notion de « déchets du bout du monde » [5][5]Expression empruntée à Romain Garcier et Laurence Rocher lors..., tel que Myra Hird (2017) le démontre dans le cas des communautés Inuïtes, ces derniers objectant les politiques modernes de gestion des déchets. À Singapour, où le système est techno-centré au travers d'installations d'incinération et de recyclage, les résultats sont également mitigés : « si l'objectif est de ne plus encombrer le territoire par des déchets en les valorisant au maximum, il semble atteint. Si en revanche on considère celui de la durabilité globale du système, c'est nettement moins le cas [du fait de l'impact environnemental de l'incinération] » (Bocquet, 2013, p. 9). De plus, dans un contexte extrême de catastrophe naturelle, la résilience des îles est mise à mal par la gestion des déchets post-catastrophe (Durand, Popescu, D’Ercole, 2015).

14Les spécificités insulaires nous ont conduits à identifier le concept de métabolisme territorial comme révélateur des contraintes insulaires et des défis transitionnels à relever. Les vulnérabilités insulaires sont donc au cœur de ces enjeux métaboliques.

\section{Comment aborder les vulnérabilités insulaires dans une perspective métabolique?}

$\underline{15}$ Cette recherche tend à développer une approche réflexive des métabolismes territoriaux pour interroger la socio-matérialité des îles et apporter une contribution théorique par le biais de la proposition de la notion de vulnérabilités métaboliques. Cette notion est une extension des vulnérabilités environnementales et territoriales (Durand, Metzger, 2009 ; Mathis et alii, 2016), issues des réflexions interdisciplinaires relatives aux territoires durables et résilients (Mathieu, 2006). Une mauvaise maîtrise des flux peut ainsi constituer un risque pour le fonctionnement même de la société à une échelle donnée, d'un point de vue sanitaire et environnemental, mais également économique et social (Durand, 2018). Le degré de vulnérabilité des territoires est parfois défini selon leur exposition aux risques produits par les déchets (Oppio, Corsi, 2017). Les travaux sur les vulnérabilités environnementales ou écologiques se sont ainsi concentrés sur les risques environnementaux liés aux infrastructures, les nuisances environnementales en lien avec des seuils d'exposition à des pollutions, et sur le manque de prise en compte de ces risques dans les politiques environnementales et urbaines (Martinez-Alier, Temper, Demaria, 2016).

$\underline{16}$ Avec la vulnérabilité métabolique, il s'agit de s'intéresser à l'extraction, la production et la circulation des ressources qui est elle-même un fort vecteur d'inégalités socio-spatiales, que ce soit à l'échelle mondiale ou locale comme en attestent les territoires miniers ou d'enfouissement des déchets, stigmates de l'anthropocène. Le territoire peut alors être considéré comme vulnérable (D'Ercole, Metzger, 2009) au titre de la remise en cause potentielle de ses modes de fonctionnement, d'approvisionnements, de gestion de ses flux et de ses rejets. Ainsi, les infrastructures articulent des circuits de différents flux qui produisent 
des vulnérabilités territoriales, matérielles et sociales (Duro, Schaffartzik, Krausmann, 2018 ; McFarlane, 2013). La notion de vulnérabilité métabolique a pour ambition de contribuer à rendre compte de la complexité des rapports de forces et de pouvoir entre territoires insulaires et territoires d'approvisionnement et de rejets. La problématique centrale de ce texte est donc la suivante : en quoi la connaissance de la circulation des flux de matière et d'énergie qui traverse un territoire insulaire permet-elle de mieux saisir les vulnérabilités qui en résultent? Nous pouvons faire l'hypothèse que les registres de gouvernance des flux sont très différents et dépendent autant de critères relationnels que territoriaux. En effet, les échelles des politiques du métabolisme urbain sont à géométrie variable selon les acteurs (Bahers, Durand, Beraud, 2017 ; Bahers, Giacchè, 2019). En cela, le contexte des PEID constitue un cas empirique qui exacerbe les relations socio-matérielles avec les territoires partenaires, régies par des mécanismes commerciaux et stratégiques de contrôle des flux de ressources, mais également par une adaptation sociale des habitants. Cette perspective de lecture sociale et spatiale de la vulnérabilité métabolique permettrait d'enrichir la réflexion sur la transition durable, en promouvant une meilleure compréhension des processus sociaux à l'œuvre (vulnérabilité, résilience, transition socio-écologique, etc.), dans une perspective de recherches opérationnelles (ingénierie urbaine et écologique).

\section{Quelle transition socio-écologique pour Ndzuwani ?}

17Proposer une lecture sociale et spatiale de la vulnérabilité métabolique dans le contexte des PEID nécessite de l'appliquer à un territoire à fort enjeu et de développer une méthodologie nouvelle. Celle-ci est présentée dans les trois sections qui suivent, à travers les outils d'analyse des flux, la démarche d'enquête et les stratégies de contournement et d'hybridation pour la mener jusqu'à son terme.

\section{Entre isolement et interconnexions : la situation de Ndzuwani}

$\underline{18}$ L'Union des Comores est l'un des PEID rassemblant des enjeux de vulnérabilité et d'adaptation aux effets du changement climatique (Bouchard et al., 2011). L'archipel volcanique des Comores, ancienne colonie française, est situé dans le canal du Mozambique entre la côte swahilie d'Afrique orientale et Madagascar (figure 1). Archipel composé de quatre îles - Anjouan (Ndzuwani), Grande Comore (Ngazidja), Mohéli (Mwali), Mayotte (Maoré) dont seule la dernière ne fait pas partie de l'Union des Comores, créée en 1975 (Taglioni, 2008). Il est à noter que l'Union des Comores telle que reconnue par les Nations Unies en 1975 comporte les quatre îles de l'archipel. Ainsi, pour le gouvernement des Comores, Mayotte est occupée par l'ancienne puissance coloniale française, malgré le dernier vote mahorais de 2011, favorable à $95 \%$ à la départementalisation française.

Figure 1 Carte de localisation de l'archipel des Comores 


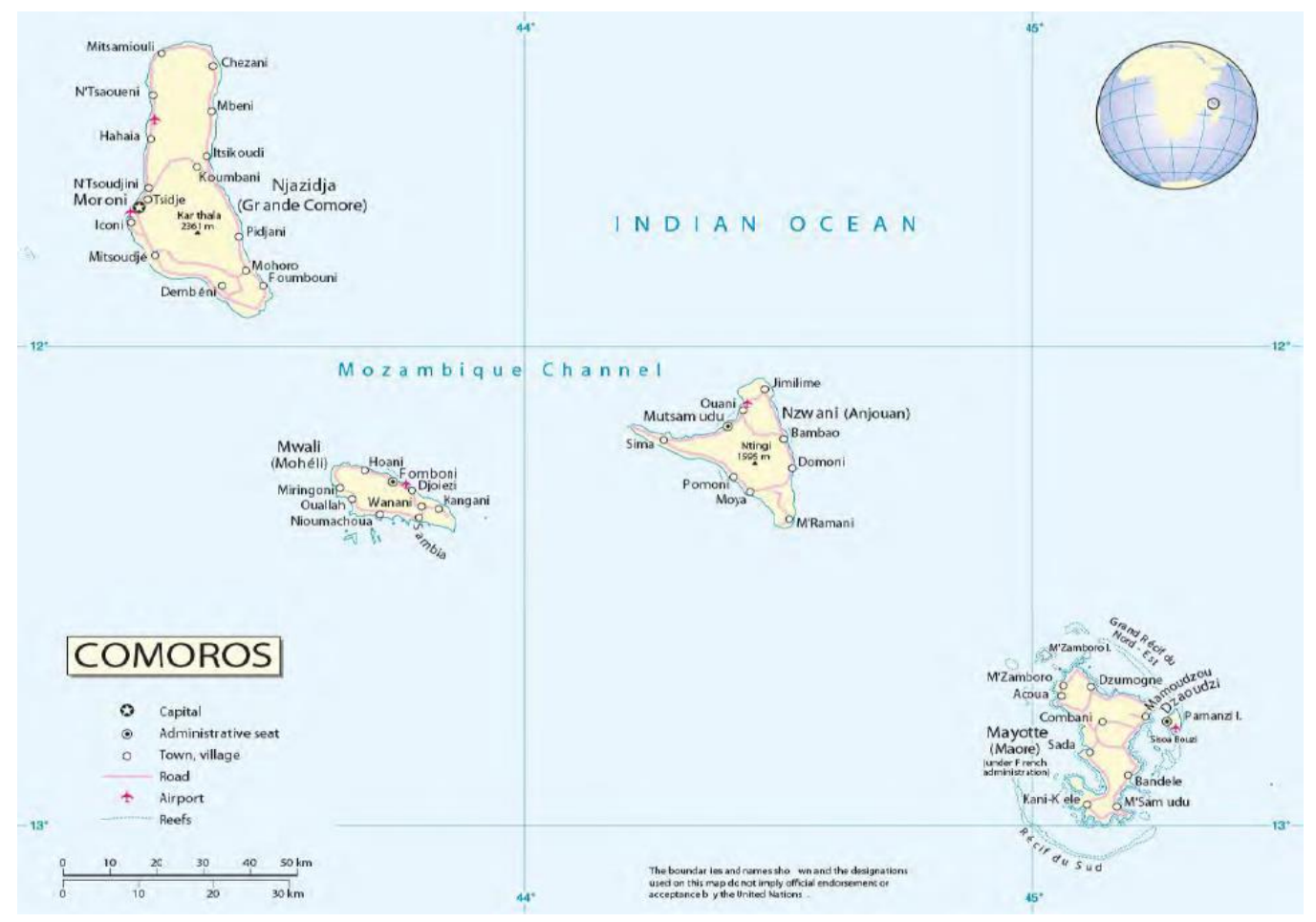

Source : ONU (Department of Peacekeeping Operations Cartographic Section), 2004.

19Ndzuwani est une île au relief très montagneux qui contraint l'habitabilité du territoire. Sa capitale, Mutsamudu, est également son seul port de marchandise. La population de Ndzuwani est d'environ 332466 habitants en 2015 (estimations de l'ONU). Elle a une superficie $\left(424 \mathrm{~km}^{2}\right)$, ce qui en fait l'île des Comores la plus densément peuplée (784 $\mathrm{hab} / \mathrm{km}^{2}$ ). Elle est également l'île la plus pauvre de l'archipel d'après la Banque mondiale (Belghith et alii, 2018) et la plus proche de Mayotte, ce qui en fait un territoire de prédilection de départ des clandestins (Taglioni, 2008).

20La situation politique aux Comores semblait s'être pacifiée jusqu'à 2018 où des réformes récentes sur la présidence tournante et les durées des mandats ont à nouveau déstabilisé la géopolitique anjouanaise. Par ailleurs, les relations avec Mayotte sont toujours extrêmement tendues du fait des difficiles migrations et des relations familiales étroites entre Mahorais et Comoriens. Par ailleurs, la balance commerciale est déficitaire pour l'Union des Comores avec Mayotte. Des chercheurs ont montré que cette dernière était aussi bien mieux préparée aux submersions marines liées aux changements climatiques (Wu-Tiu-Yen, 2015) et aux catastrophes naturelles (Legoff, 2011) que le reste de l'archipel.

21Les déficits structurels de l'Union des Comores sont nombreux dans les secteurs de la santé, de l'éducation, des infrastructures, des transports et du tourisme, en partie en raison d'un manque d'investissement de fonds publics et privés. Comme l'explique Taglioni (2008) : «L'histoire et la géographie se sont conjuguées pour placer cet archipel en marge du développement politique, économique et des flux mondiaux » (p. 1). Cela se traduit également par un fort taux de pauvreté et de mortalité infantile, une alphabétisation faible et une espérance de vie limitée. Pour autant, la criminalité est très faible et la quiétude règne dans les rues de Mustamudu. 
22L'agriculture est le secteur économique le plus développé aux Comores (environ $40 \%$ du PIB). Elle associe agriculture vivrière traditionnelle (Robineau, 1966) et cultures de rentes comme la vanille, l'ylang-ylang et le clou de girofle, dédiées à l'export et contrôlées par des communautés locales issues d'un héritage commercial (Blanchy, 2015). Ainsi, la population est majoritairement rurale (environ $70 \%$ ) mais tend à s'urbaniser dans les villes littorales (Gérard, 2009).

23Malheureusement, les paysages très riches de ces îles sont menacés par la déforestation, les activités humaines (Sinane et alii, 2011) et les dépôts de déchets sauvages (photo $\mathrm{n}^{\circ} 1$ ). Ces derniers sont souvent jetés dans les mers ou rivières, parfois brûlés (photo $\mathrm{n}^{\circ} 2$ ). D'autres sont enfouis dans des sites inadaptés, ce qui n'a pas manqué de déclencher des conflits sociaux dans l'île (photo $\mathrm{n}^{\circ} 3$ ).

Photo $n^{\circ} 1$ Le rejet de déchets sur la plage de Mutsamudu Source : Perez, 2018.

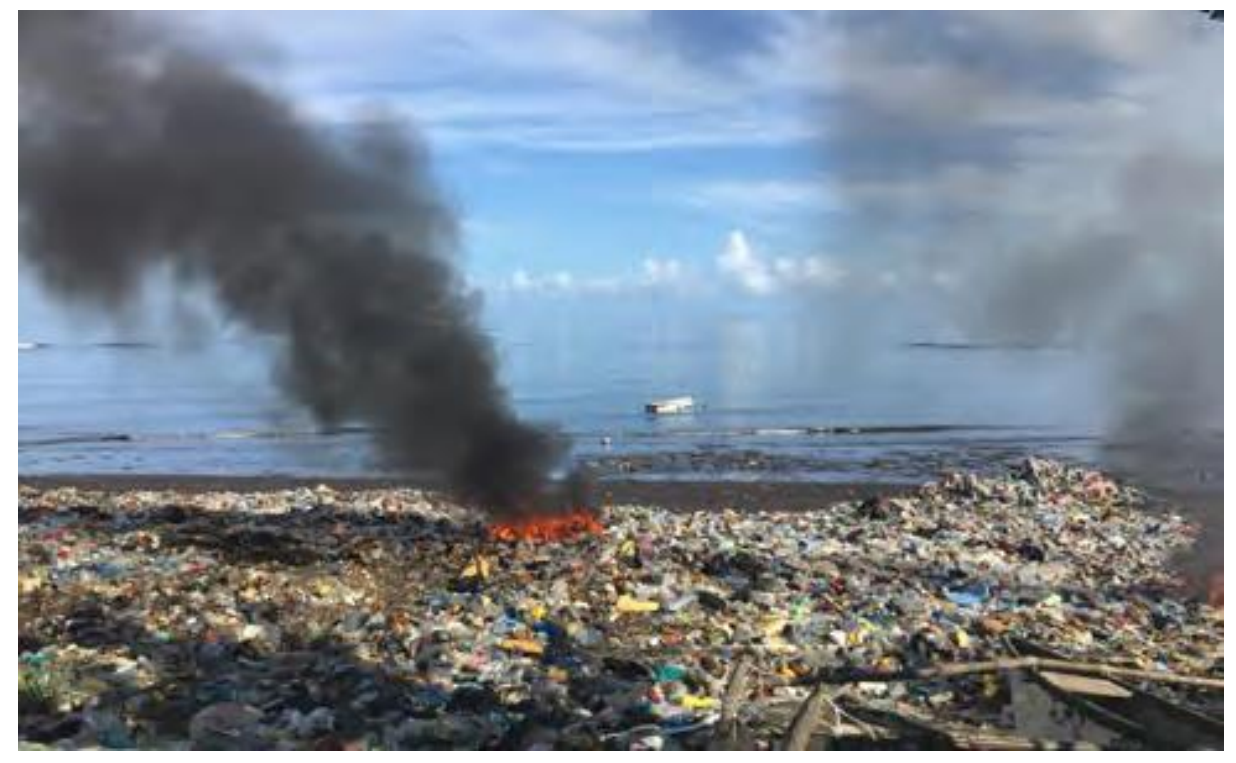

Photo $n^{\circ} 2$ Absence de contrôle de la décharge « contrôlée » de Domoni Source : Bahers, 2018. 


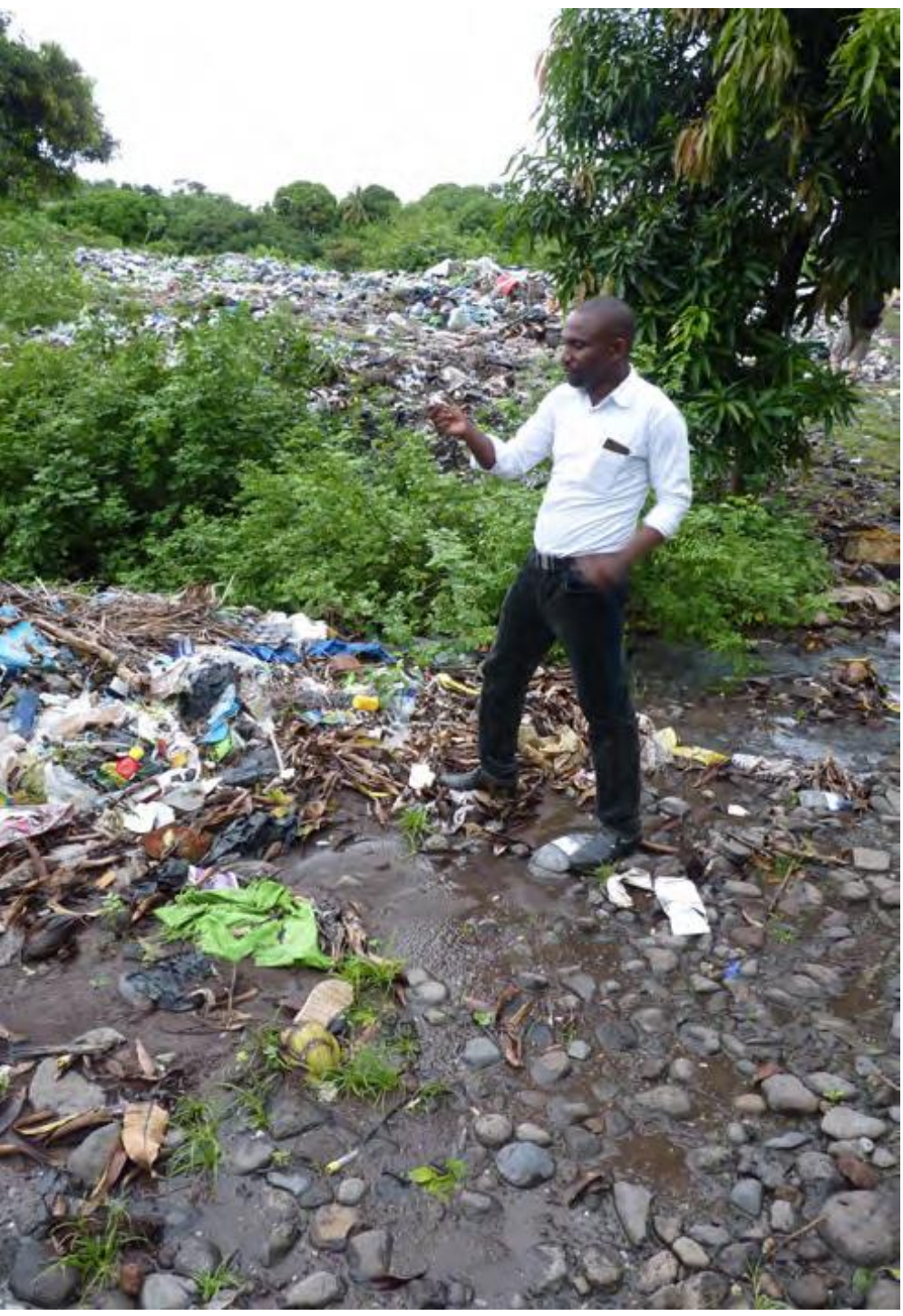

Photo $n^{\circ} 3$ Opposition à la construction d'une décharge dans la commune de Ouani Source : Perez, 2018.

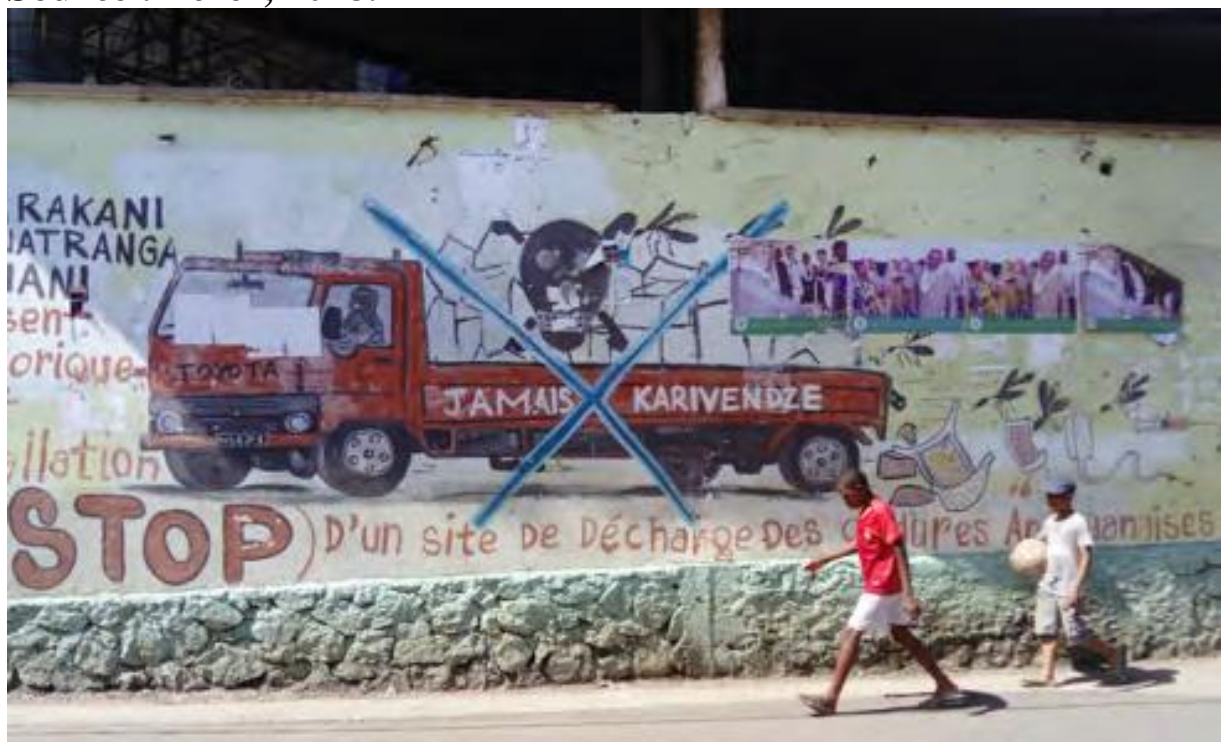


$\underline{24 C e t t e ~ s i t u a t i o n ~ p o u s s e ~ l e ~ g o u v e r n e m e n t ~ d e ~ l ' U n i o n ~ d e s ~ C o m o r e s ~ e t ~ l e ~ g o u v e r n o r a t ~ d e ~}$ Ndzuwani à s'impliquer dans une transition vers une économie raisonnée des ressources (notamment du bois forestier) et une gestion plus durable des déchets. Ainsi, les Comores se dotent progressivement de législations visant à responsabiliser les collectivités pour la collecte et le traitement des déchets, à limiter l'exploitation du bois forestier par des quotas, à se doter d'un cadastre foncier pour aménager le territoire et d'infrastructures pour l'irriguer. Ces politiques locales connaissent des difficultés à être appliquées mais les termes d'une transition vers une économie plus résiliente et durable sont au cœur de la stratégie locale de développement.

\section{Une analyse des flux sous contraintes : informalité et manque de statistiques}

25L'île de Ndzuwani se prête donc bien au développement d'une méthodologie de compréhension du métabolisme territorial à l'échelle insulaire. Deux défis sont rapidement apparus face à cette ambition : l'importance des activités informelles dans les filières économiques et le manque de bases de données statistiques consolidées et complètes.

26Nous utilisons une AFM (Analyse des Flux de Matières) qui fournit des informations globales sur les caractéristiques physiques des économies nationales, basée sur l'adaptation de la méthode du service européen de la statistique (EUROSTAT, 2009). Elle permet d'identifier les principaux flux entrants et sortants d'un territoire et ainsi d'en mesurer la matérialité et les conséquences en termes de pressions sur l'environnement. Ces dernières années, un nombre croissant d'études de cas nationales ont développé des indicateurs de flux de matériaux sur de plus longues périodes, examinant l'émergence de profils métaboliques (Krausmann et alii, 2018 ; Schandl et alii, 2018 ; Wiedenhofer et alii, 2019), produisant une recherche socioécologique sur le long terme (Singh et alii, 2013). Ce choix a également l'avantage de prolonger des études déjà réalisées (Duro, Schaffartzik, Krausmann, 2018 ; Martinico-Perez et alii, 2018) et de permettre la comparaison avec d'autres territoires insulaires investigués par la communauté scientifique (Cecchin, 2016 ; Krausmann, Richter, Eisenmenger, 2014).

27La méthode AFM permet de discuter les indicateurs de flux matériels directs, tels que l'extraction intérieure (DE : Domestic extraction), les importations et exportations, les entrées matérielles (DMI : Domestic material input), la balance commerciale physique (PTB : Physical trade balance), les rejets vers la nature, tels que les déchets et émissions atmosphériques (DPO : Domestic processed output), l'addition au stock (NAS : Net Addition to Stock) et la consommation intérieure de matières (DMC - Domestic material consumption $=\mathrm{DE}+\mathrm{PTB})$. L'AFM fait ainsi la distinction entre de nombreux produits et marchandises qui peuvent être agrégés par catégories. Par ailleurs, notre analyse accorde une place importante aux relations entre hinterland d'approvisionnement et territoire insulaire. Certains flux sont donc étudiés de manière approfondie, notamment les flux d'importation, qui sont regroupés selon les grands types de marchandises (appareils manufacturés, produits agricoles et alimentaires, matériaux de construction, minerais et produits métallurgiques, combustibles et produits pétroliers, divers).

28Les études internationales de métabolisme socio-économique utilisent le plus souvent des sources de données statistiques nationales et internationales et des procédures d'estimation standard pour les flux non couverts par les statistiques (Fischer-Kowalski et alii, 2011 ; 
Krausmann et alii, 2009). Dans notre cas, les statistiques ne couvrent pas l'ensemble des flux, ce qui nous a amenés à développer des stratégies de contournement et à mobiliser une expertise locale lors d'une enquête à plusieurs phases. Cependant, certains indicateurs n'ont pas été calculés (tels que l'extraction inutilisée, les flux indirects et les flux d'équilibrage), du fait du manque de données fiables, ce qui est le cas pour de nombreuses études de métabolismes urbains. Cette limite méthodologique n'empêche pas d'étudier d'autres aspects du métabolisme insulaire.

\section{Déroulé de l'enquête en deux étapes : cadrage commun et observation in situ}

29Nous [6][6]Travail de recherche mené dans le cadre d'un partenariat entre... avons effectué une mission sur place pour identifier les principaux flux avec une quarantaine d'acteurs institutionnels comoriens (douanes, port), anjouanais (directeurs économique et environnement du Gouvernorat de Ndzuwani, ministres locaux en charge de l'environnement, de l'économie, de l'urbanisme), communaux (élus locaux), associations environnementales (Ouani vert, Commission Mutsamudu Propre, Sac Marie) et ONG internationales (Initiative Développement, Naipenda Comores, Gevalor). Les données des douanes ont permis d'avoir une estimation précise des échanges de flux avec l'extérieur de l'île. Les dires d'acteurs nous ont également permis d'affiner la compréhension des flux visibles et moins visibles qui circulent sur l'île. Nous avons donc pu construire une représentation qualitative du métabolisme insulaire, qui fut la piste principale de notre enquête. Ce parti-pris méthodologique représente une originalité dans le champ des études du métabolisme territorial. Environ quatre-vingt matières furent recensées par les acteurs pendant cette mission.

30La deuxième étape fut une observation sur le terrain plus longue des pratiques des acteurs de la circulation des flux (Perez, 2018). Une quarantaine d'acteurs furent rencontrés et de nombreuses situations métaboliques furent observées (extraction de matières de construction, importations-exportations sur le port, usages matériels, production de déchets, recyclage ou dépôt sauvage). Pour certaines de ces activités, l'informalité représente une part importante du marché. Ce fut un des enjeux principaux à surmonter lors de l'enquête. Pour lever ce verrou, des visites de terrain ex abrupto ont été réalisées afin de saisir la réalité des opérations.

\section{Méthodologie ascendante et filières informelles : vers une hybridation des données}

$\underline{31} L e$ peu de données statistiques existantes est établi à l'échelle nationale alors que seule l'île de Ndzuwani, qui constitue l'une des trois îles de l'Union des Comores, nous intéresse. Le déroulement méthodologique est donc en partie inspiré d'une démarche ascendante, c'est-àdire qui intègre les acteurs à l'enquête et donc à l'identification des flux, ce qui fut très peu développée dans la littérature comme le suggèrent les états de l'art de Baynes et Wiedmann (2012) et Chrysoulakis et alii (2013). Cette démarche située s'intéresse aux pratiques quotidiennes et aux représentations des acteurs (Lawhon, Ernston, Silver, 2014) et donc à 
l'étude d'une AFM plus proche des situations rencontrées au sein du territoire. La pratique informelle est également un défi méthodologique d'importance, et très peu d'AFM s'y intéressent (Guibrunet et alii, 2016). L'économie informelle peut s'appréhender ici sous plusieurs angles socio-professionnels. Elle représente un ensemble d'acteurs indispensables au fonctionnement des territoires (McFarlane, 2012) et des filières institutionnalisées, comme la gestion des déchets ou la construction, pour lesquelles l'articulation avec le secteur informel permettrait la fourniture d'un service plus efficace (Durand, De Oliveira Neves, 2019 ; Kakeu-Tardy, 2018). Il s'agit là d'un enjeu de formalisation du secteur informel ou d'une gestion partagée. Cependant, l'informalité peut aussi représenter une pratique engagée politiquement, contestataire, voire subversive (Monsaingeon, 2017). En cela, le secteur informel ouvre de nouvelles voies de pratiques entre les acteurs et les matières. La consistance sociale et environnementale de l'informel est donc en évolution dans le champ des sciences sociales vers une prise en compte de son rôle et de son utilité, à laquelle nous nous rallions dans la méthodologie.

32Concernant l'extraction intérieure, c'est-à-dire la production locale de produits, matériaux et énergie, nous avons combiné des sources de terrain, des recherches géomatiques sur Ndzuwani (Boussougou, Brou, Mohamed, 2015), et des bases de données internationales, notamment de la FAO (FAOSTAT, 2018). Par ailleurs, certaines sources internationales se sont avérées largement incomplètes. Alors que la principale base de données utilisée par les chercheurs sur le métabolisme pour l'extraction minière est la « U.S. Geological Survey (USGS) », la dernière publication (USGS, 2010) ne mentionne aucune production locale de matériaux à Ndzuwani. Pourtant, il existe deux carrières autorisées qui produisent environ $80 \%$ de minéraux (sable de différentes granulométries, gravier, cailloux) extraits du territoire insulaire. En outre, sont présentes des carrières informelles qui ont un rôle important dans 1'approvisionnement local. La connaissance empirique nous a amenés également à observer des pratiques informelles de prélèvements de sables dans les littoraux et rivières. À partir d'observations répétitives des pratiques et des entretiens auprès des acteurs, nous avons estimé les quantités extraites. D'autres recherches (Sinane et alii, 2011) nous ont permis de croiser les résultats afin de vérifier leur fiabilité. Il est néanmoins impossible d'obtenir la complétude des données concernant les filières informelles, mais cette démarche reste plus convenable que celle qui consiste à se référer seulement aux bases de données internationales qui omettent en grande partie la factualité du fonctionnement territorial. Il en est de même pour la production de bois qui est essentiellement une activité informelle, pour laquelle nous avons mobilisé les cartes d'occupation des sols (Boussougou, Brou, Mohamed, 2015). L'usage du bois concerne la cuisson domestique, la construction et la distillation pour alimenter les alambics.

33En ce qui concerne les rejets, les sources sont difficiles à identifier. Les déchets ménagers sont peu gérés par la collectivité, ce qui ne permet pas d'avoir des relevés quantitatifs réguliers. Les déchets sont le plus souvent jetés dans les rivières ou sur les littoraux, enfouis ou brûlés. D'après une étude récente empirique (Fouquet, 2014), les Anjouanais produiraient environ $0,4 \mathrm{~kg} / \mathrm{hab} / \mathrm{jour}$ de déchets ménagers, dont $80 \%$ de déchets organiques. Le recyclage des déchets ménagers et industriels est quasiment inexistant étant donné qu'il n'y a pas d'infrastructures de tri et de traitement des déchets sur l'île. On peut néanmoins noter quelques initiatives informelles d'opérateurs qui récupèrent de l'aluminium pour faire des marmites ou d'éleveurs qui collectent des déchets organiques pour l'alimentation de volailles. Cependant, les plastiques, ferrailles, véhicules, textiles, déchets médicaux, emballages sont jetés sur les plages et dans les zones de dépôts sauvages, alors que ce sont des matières de récupération qui pourraient être valorisées sur place ou exportées vers un marché à 
1'international. En revanche, les déchets de construction sont très souvent réutilisés pour des remblais, voire des constructions neuves. Les déchets de l'agriculture ont été estimés en croisant les données issues de la FAO (FAOSTAT, 2018) et le nombre de bovins et ovins. Les effluents sont généralement épandus sur les sols, ce qui est considéré par la méthode AFM comme un usage dissipatif.

34Cette hybridation des données empiriques et de bases de données internationales nous a permis de lever les verrous liés à l'informalité et au manque de statistiques nationales. Quoiqu'imprécise, cela conduit à une représentation métabolique de l'île de Ndzuwani et à produire une connaissance d'ensemble de la circulation des flux de matières et d'énergie.

\section{Les dimensions matérielles, sociales et environnementales d'un métabolisme insulaire}

35La méthodologie développée permet d'identifier les différentes dimensions du métabolisme insulaire de Ndzuwani. Il s'agit tout d'abord de la matérialité du métabolisme : la circulation des flux est ainsi présentée, depuis l'extraction des matières, jusqu'à leur fin de vie. Ensuite, une lecture spatiale de ce métabolisme conduit à interroger les relations avec les territoires d'approvisionnement. La dernière section s'attache à décrire des situations de vulnérabilités métaboliques découlant des conséquences sociales et environnementales de la circulation des flux.

\section{Les principaux flux de matière à Ndzuwani : extraction locale, importations, consommation et rejets}

$\underline{36} \mathrm{La}$ figure 2 synthétise les résultats obtenus pour l'île de Ndzuwani pour l'année 2016. Il est à noter que le poids des importations est important (27\% de la DMI), mais pas majoritaire par rapport aux extractions locales (73\% de la DMI), contrairement à ce que l'on aurait pu penser intuitivement. Les importations concernent environ $0,4 \mathrm{t} / \mathrm{hab} / \mathrm{an}$ de matières et d'énergie, notamment les matériaux de construction (ciment, chaux ou plâtre), qui représentent $51 \mathrm{kt}$ en 2016 (soit $40 \%$ des importations) ; chiffre important pour des marchandises pondéreuses et du même ordre de grandeur que celui de l'extraction locale $(84 \mathrm{kt})$. Cela montre la difficulté à s'approvisionner localement, car pour ces marchandises, le coût du transport, même maritime, est majeur.

Figure 2 Bilan du métabolisme insulaire 


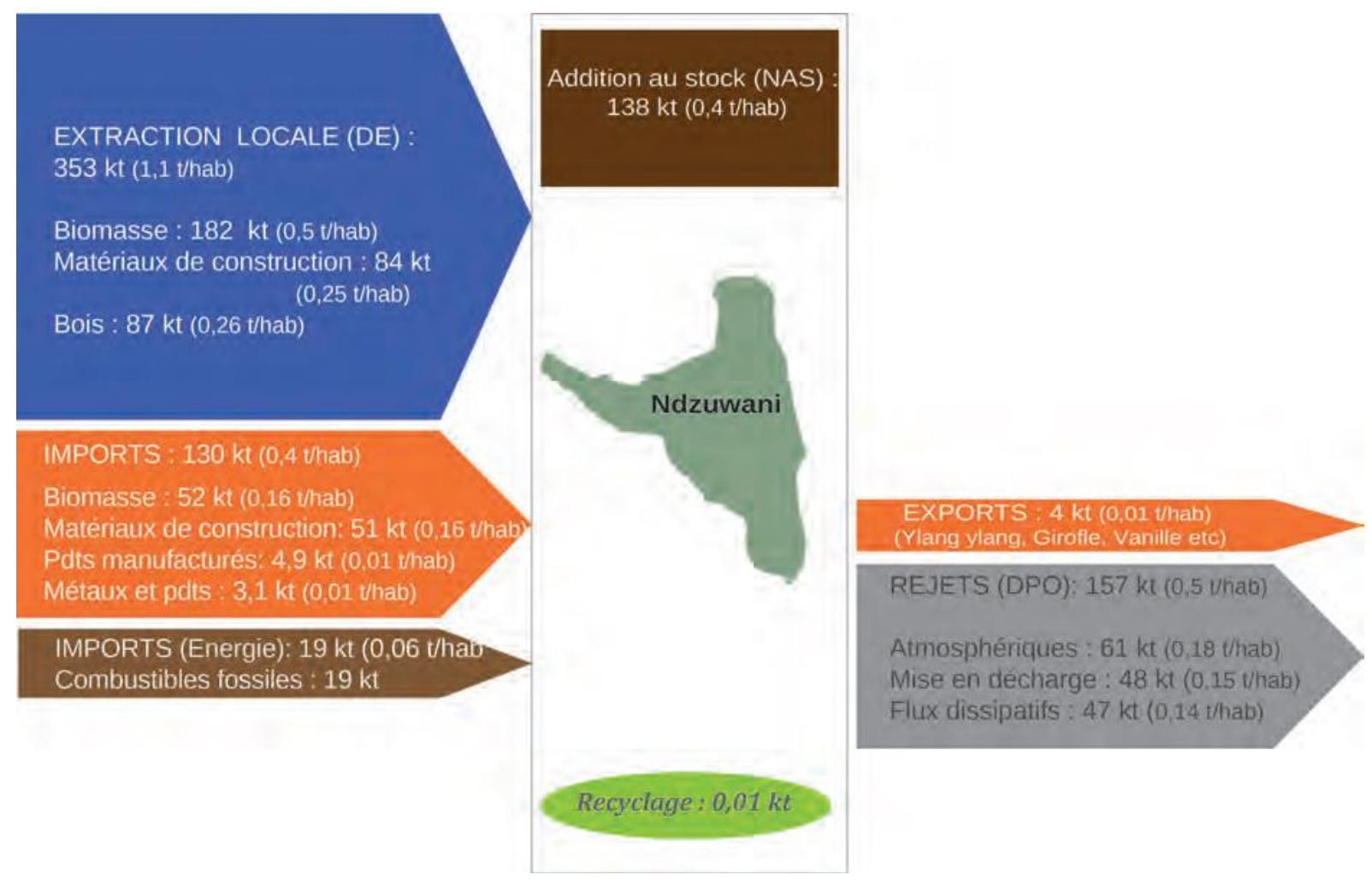

Réalisation : Bahers, 2018.

37L'extraction locale (DE) est constituée à plus de la moitié par la biomasse, c'est-à-dire les cultures vivrières. Il s'agit d'une production de $0,55 \mathrm{t} / \mathrm{hab} /$ an directement autoconsommée complétée par 0,16 t/hab d'importations de produits alimentaires. Les matériaux de construction et le bois complètent cette extraction. Pour autant, il est difficile d'envisager à partir de ces flux une piste d'autosuffisance insulaire, car en l'état une partie de l'extraction du sable se fait au détriment des littoraux et la demande en bois met en danger la pérennité des forêts insulaires. Par ailleurs, les exportations sont directement liées à cette extraction intérieure, car, pour produire les quelques marchandises vendues outre-mer, il faut de grandes quantités de bois pour alimenter les alambics (utilisés pour la distillation d'Ylang-Ylang). Il apparaît ainsi une forte dépendance entre ces deux grandes catégories de flux, que l'on pourrait envisager en termes de liens métaboliques entre hinterland et territoires de consommations (Barles, 2017 ; Marty, 2013).

38Dans la consommation intérieure (DMC) - toutes marchandises confondues -, la part de la biomasse est la plus importante (48\%), suivie par les matériaux de construction (28\%) et les combustibles fossiles (22\%). Les appareils manufacturés et les produits métallurgiques représentent une très faible part dans la consommation, ce qui explique aussi le peu d'équipements en biens durables (équipements ménagers, voitures, produits électroniques) des Anjouanais. L'augmentation de la consommation de ces équipements est prégnante à Ndzuwani, mais elle est très loin de se rapprocher de celle des habitants des pays du Nord global. En cela, l'addition annuelle au stock, c'est-à-dire les matières qui restent sur le territoire, est constituée principalement de matériaux de construction et représente environ $138 \mathrm{kt} / \mathrm{an}$, donc $0,42 \mathrm{t} / \mathrm{hab}$. 
39La DPO (rejets vers la nature) est constituée de rejets atmosphériques liés à la combustion des énergies fossiles, du bois et de déchets enfouis ou épandus, principalement organiques. Il est à noter que contrairement à d'autres pays exportateurs de ressources, peu de déchets résultent de l'extraction et de la fabrication des produits exportés, même si les eaux usées issues de la production d'huiles essentielles sont importantes (Tongnuanchan, Benjakul, 2014). Enfin, le recyclage est quasi inexistant par rapport à la production de déchets insulaires.

\section{Lecture spatiale des flux anjouanais : fortes importations à travers le monde et faible externalisation des rejets}

40Si l'on approfondit la spatialisation du métabolisme territorial, on peut développer deux résultats principaux concernant les rejets et les approvisionnements.

41Tout d'abord, les rejets ne sont pas du tout externalisés à Ndzuwani, à l'opposé des territoires européens ou nord-américains qui en exportent certaines catégories. Ndzuwani ne bénéficie pas de la possibilité financière et technique d'externalisation de ces matières et internalise donc le traitement de l'ensemble de ses déchets. Ainsi, il s'agit d'une conséquence négative bien visible, ce qui pourrait paradoxalement engendrer une prise en charge plus transparente et contrôlée par le territoire. Pour autant, le manque d'infrastructures de collecte et de traitement des déchets et le manque de structuration des pouvoirs publics en font un véritable obstacle à une bonne gestion.

42Par ailleurs, les aires d'approvisionnement des marchandises sont localisées sur l'ensemble de la planète (cf. figure 3). Persistent toutefois des relations importantes avec les pays ou territoires voisins. Le répertoire des douanes différencie par exemple les importations qui viennent de Mayotte, de la Réunion et de la France métropolitaine, du fait du contexte géopolitique et de l'importance persistante des flux entre ces territoires. Ndzuwani est un important récepteur des matières exportées de Mayotte, notamment des produits manufacturés (réfrigérateurs, machines à laver, fenêtres, vaisselle, textiles, etc.), des combustibles fossiles, et des eaux minérales. Ces flux, qui suivent la trajectoire inverse des migrations, montrent les relations commerciales fortes entre les deux îles de l'archipel, au-delà de la lecture classique de contentieux politiques entre elles. Les conséquences en termes de métabolisme de cette situation prouvent que des voies de mutualisation de flux sont envisageables et qu'une mise en politique des flux (Garcier, Martinais, Rocher, 2017) pourrait être à l'œuvre.

Figure 3 Spatialisation du métabolisme : fortes importations à travers le monde 


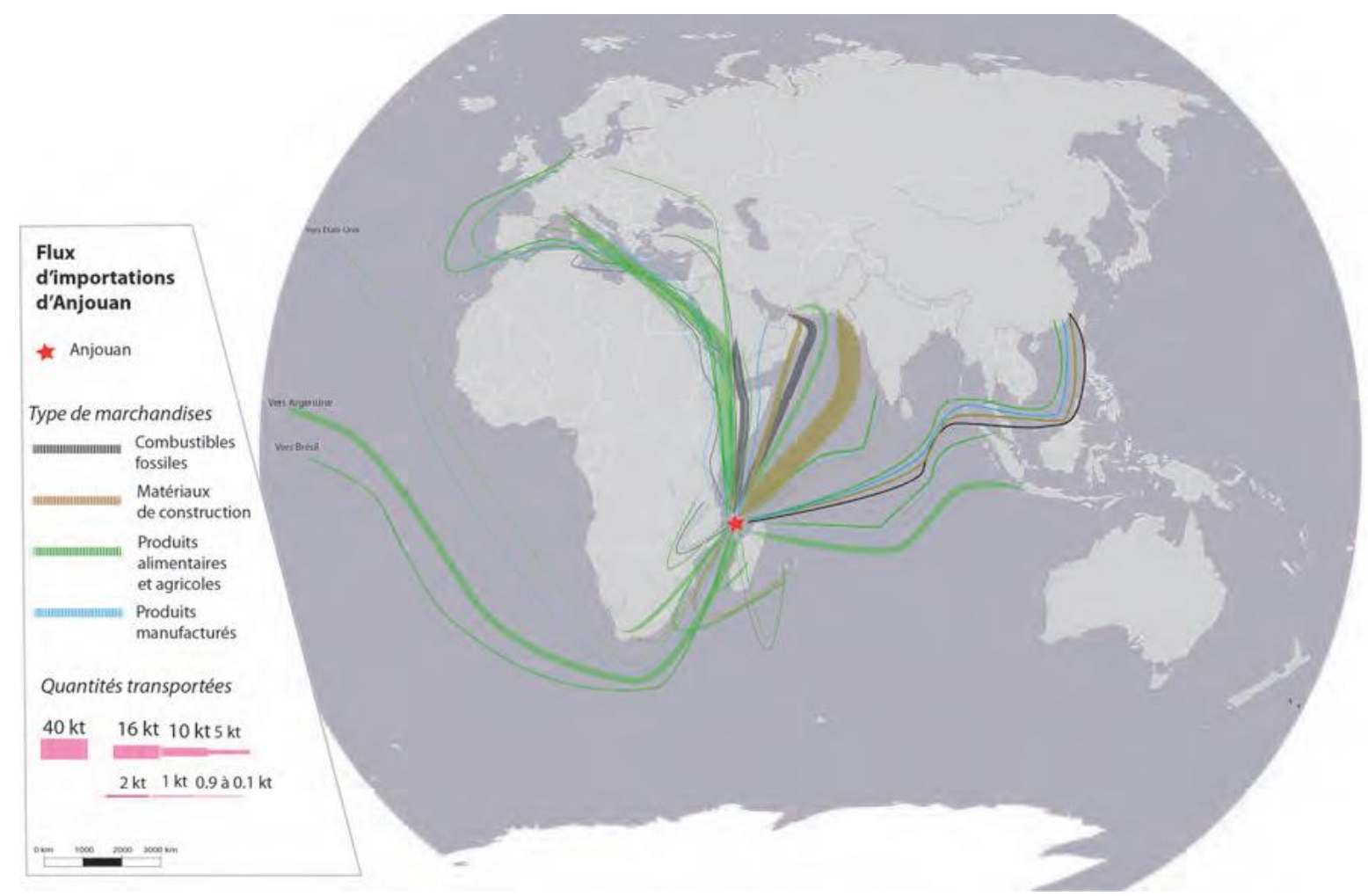

Source : Données du port de Mutsamudu, 2016. Réalisation : Bahers, 2018.

43Si la France est le premier partenaire de Ndzuwani, les Émirats Arabes Unis sont les deuxièmes car ils tiennent le rôle de nouveau emporium (Wœssner, 2016), c'est-à-dire un pôle d'attraction et de redistribution pour les marchandises, avec la panoplie de services urbains modernes. Cette place devenue forte est aussi le symbole de la croissance exponentielle des transports maritimes. Concernant les combustibles fossiles, on retrouve les circuits classiques de transport maritime depuis les pays pétroliers. La Chine est le troisième fournisseur de Ndzuwani, notamment en produits manufacturés. Les relations avec les territoires proches se réalisent via Mayotte, la Tanzanie, ainsi que la Réunion. Les produits alimentaires ont des trajectoires spatiales beaucoup plus variées. Trente-six territoires approvisionnent Ndzuwani (Danemark, Espagne, Maroc, etc.). Le riz et les produits carnés sont les principales marchandises alimentaires importées. L'Afrique du Sud, distante d'environ $3000 \mathrm{~km}$, est identifiée comme ayant de fortes relations commerciales avec les Comores, notamment pour les équipements électriques et électroniques. D'autres îles de l'océan indien, comme Maurice et Madagascar, sont des fournisseurs importants de marchandises alimentaires. Cet espace pourrait d'ailleurs être envisagé comme une échelle de proximité pour de nombreuses marchandises et amener à une réflexion sur l'inter-insularité des flux. Les appareils manufacturés, qui sont importés en quantité assez faible (10 kg/hab/an), proviennent des pays asiatiques comme la Chine et l'Inde, et du Moyen-Orient (Émirats Arabes Unis et Arabie Saoudite). Concernant les matériaux de construction, le Pakistan est le plus important partenaire de Ndzuwani, par le biais de l'approvisionnement en ciment (40 000 t/an). De manière moins substantielle, la Chine, les Émirats Arabes Unis et la Turquie sont également des fournisseurs de ces matières.

44Ndzuwani est donc bien intégrée à des circuits de mondialisation des marchandises (Blanchy, 2015), et entretient des relations fortes avec des territoires voisins, notamment à l'échelle des îles de l'océan indien. Cet espace pourrait être envisagé pour de nombreuses 
mutualisations infrastructurelles, notamment pour l'approvisionnement en matières alimentaires ou en matériaux de construction, et pour la gestion des déchets et des ressources primaires, du fait de plus faibles distances et de métabolismes probablement très proches entre ces îles. Pour autant, ce sont bien ces flux qui exercent une pression sur l'île de Ndzuwani et exacerbent les vulnérabilités.

\section{Vers un cadre d'analyse des vulnérabilités métaboliques : les déchets organiques, le sable et le bois}

$\underline{45}$ Certaines filières à Ndzuwani donnent à voir des conséquences sociales et environnementales de la circulation des flux. À partir de l'observation in situ du contexte territorial, trois matières (les déchets organiques, le sable et le bois) nous paraissent particulièrement symptomatiques et cristallisent des vulnérabilités insulaires.

46Tout d'abord, la spatialisation du métabolisme montre une production de $48 \mathrm{kt}$ de déchets ménagers, c'est-à-dire environ $150 \mathrm{~kg} / \mathrm{hab} / \mathrm{an}$. Le traitement des déchets est principalement internalisé dans des conditions qui renforcent les fragilités insulaires. En effet, une partie des déchets est jetée dans les rivières ou sur le littoral, après avoir été parfois brûlée sommairement aux abords des plages comme sur la baie urbanisée de Mutsamudu ( $c f$. Photo $\mathrm{n}^{\circ} 4$ ). L'autre partie est enfouie, ou plutôt entreposée en milieu rural. Si ces déchets étaient correctement pris en charge et valorisés, ils pourraient représenter un atout pour l'île. Les pouvoirs publics, ONG locales et internationales mettent en œuvre des solutions, mais ces acteurs ne partagent pas toujours les mêmes visions et les opérations restent malheureusement des signes faibles de transition. Cette gestion accentue les conflits sociaux et met également en péril de nombreux espaces littoraux et ruraux ( $c f$. Photo $\mathrm{n}^{\circ} 5$ ). Étant donné le fort taux de fermentescibles de ces déchets $(80 \%)$, il s'agit là d'une perte hypothétique pour le secteur de l'agriculture qui pourrait substituer des intrants par ces résidus organiques afin de nourrir les sols.

Photo $n^{\circ} 4$ : Dépôt de déchets brûlés au bord de la plage de Mutsamudu Source : Bahers, 2018. 


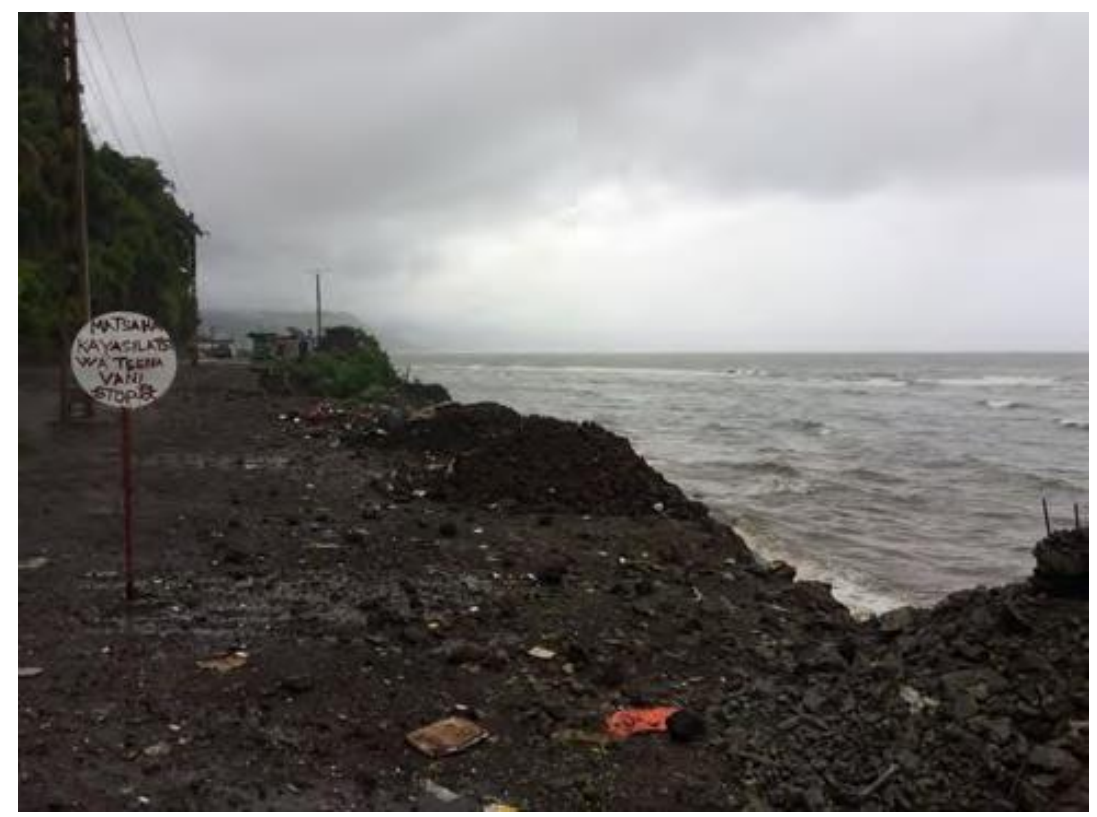

Photo $n^{\circ} 5$ : Balayeur de route qui repousse les déchets issus de la mer Source : Bahers, 2018.

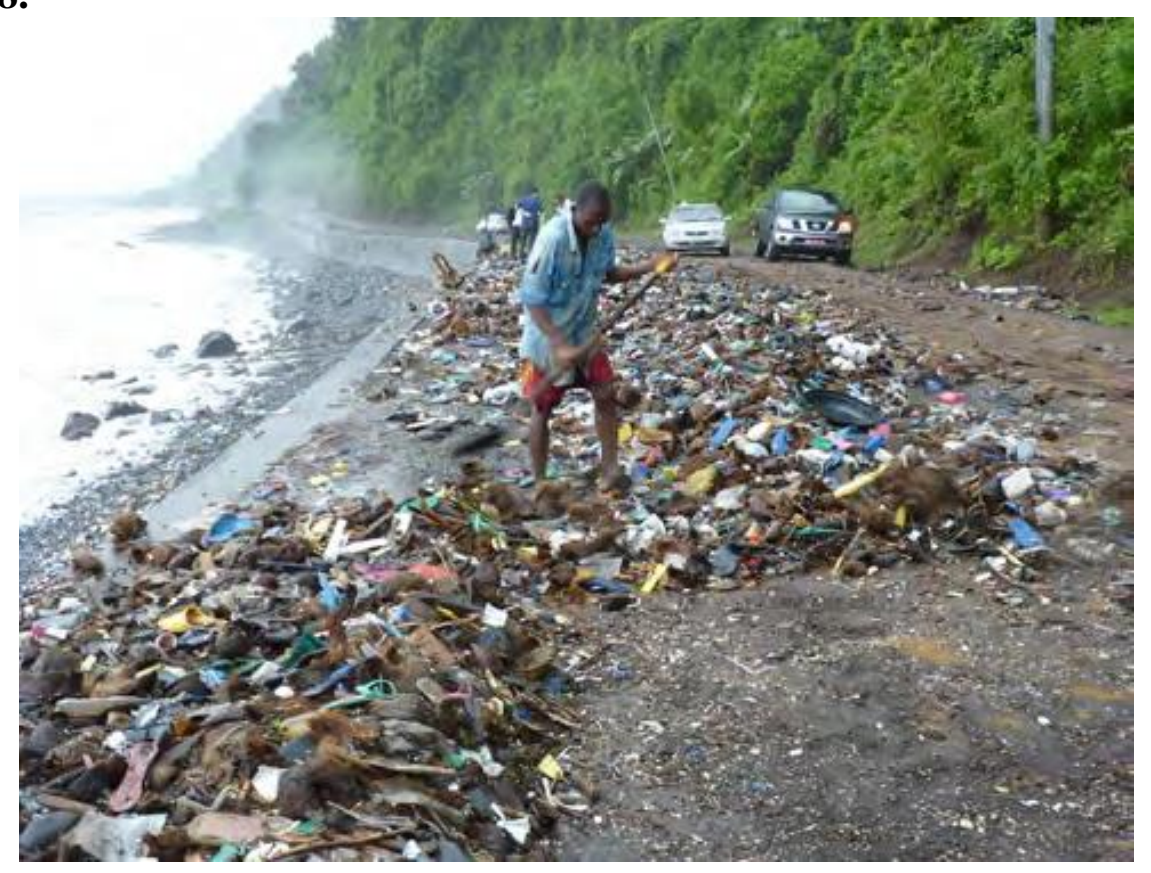

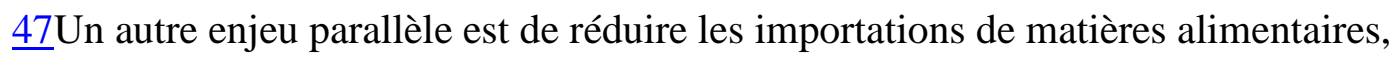
actuellement environ $160 \mathrm{~kg} / \mathrm{hab} / \mathrm{an}$, grâce à une production insulaire plus auto-suffisante. Si la production de biomasse augmente sur une temporalité de dix ans $(+65 \%)$ pour une démographie également en hausse de $30 \%$, les importations croissent de manière encore plus importante d'environ $77 \%$ selon la FAO (FAOSTAT, 2018). Ainsi, la part de la production insulaire s'affaiblit dans la consommation domestique, ce qui n'est pas une trajectoire d'autonomie alimentaire. Pour tous ces motifs, la gestion des ressources et des déchets organiques est un illustrateur des vulnérabilités métaboliques, qui articulent :

- des processus environnementaux, liés aux dépôts sauvages de déchets qui détruisent des espaces littoraux ; 
- des processus matériels, en particulier ouverture des cycles biogéochimiques et linéarisation du métabolisme ;

- des processus socio-politiques, en l'absence de prise en charge des déchets due aux déficits infrastructurels et au manque de ressources financières ;

- et des processus historiques, car l'autosuffisance de l'île est plus faible sur une échelle temporelle d'une dizaine d'années.

$\underline{48}$ Une autre situation illustrant les vulnérabilités métaboliques est la filière des matériaux de construction, en plein essor. En effet, le secteur se développe fortement avec une urbanisation grandissante sur le littoral. Les surfaces urbanisées ont ainsi doublé entre 1995 et 2014 (Boussougou, Brou, Mohamed, 2015). Il en résulte une importation de ciment, de chaux ou de plâtre très élevée en termes de volumes physiques et financiers (principalement issus du Pakistan pour $40 \mathrm{kt}$ en 2016, et dans une moindre mesure des Émirats Arabes Unies et de Chine, $c f$. figure 3). Ces importations révèlent le manque de ressources propres et le déficit d'infrastructures de construction, véritables freins au développement économique du secteur. Pour autant, depuis quelques années, des carrières contrôlées sur l'île de Ndzuwani ont été ouvertes, ayant pour conséquence de diminuer les importations. Mais les entretiens avec les acteurs ont montré que ces matériaux sont trop onéreux pour nombre de ménages. Cela a pour conséquence de ne pas diminuer l'extraction informelle de sable sur les littoraux ou dans les rivières, qui concerne $20 \%$ environ de la production insulaire de matériaux de construction en 2016. Cette pratique est très pénible sur le plan du travail humain ( $c f$. Photo $\mathrm{n}^{\circ} 6$ et 7 ) et peu rémunératrice : «J'extrais une quantité de 30 seaux par jours. Lorsque j'arrive à une quantité de $4 \mathrm{~m}^{3}$, environ 1 mois pour y arriver, je vends le sable à 50000 Frc [environ 100 Euros] » (Entretien travailleur extrayant le sable de la rivière de Ouani, 2018). Elle est également désastreuse pour l'environnement paysager de l'île (Sinane et alii, 2011), ainsi que pour les infrastructures routières le long des littoraux qui s'effondrent parfois à cause du recul de la plage ( $c f$. Photo $\mathrm{n}^{\circ} 8$ ). Malgré les interdictions réglementaires renouvelées par les autorités publiques, c'est encore $13 \%$ de la consommation des matériaux de construction qui provient de cette filière informelle. Ces procédés de construction impliquent des ouvrages parfois moins fiables et moins résistants aux aléas climatiques (notamment les inondations et les cyclones). Cette vulnérabilité métabolique s'exerce donc dans la combinaison d'une matérialité à fort impact environnemental, de contraintes sociales de travail et d'infrastructures défaillantes.

Photo $\mathbf{n}^{\circ} 6$ : Récupération de sable dans une rivière Source : Perez, 2018.

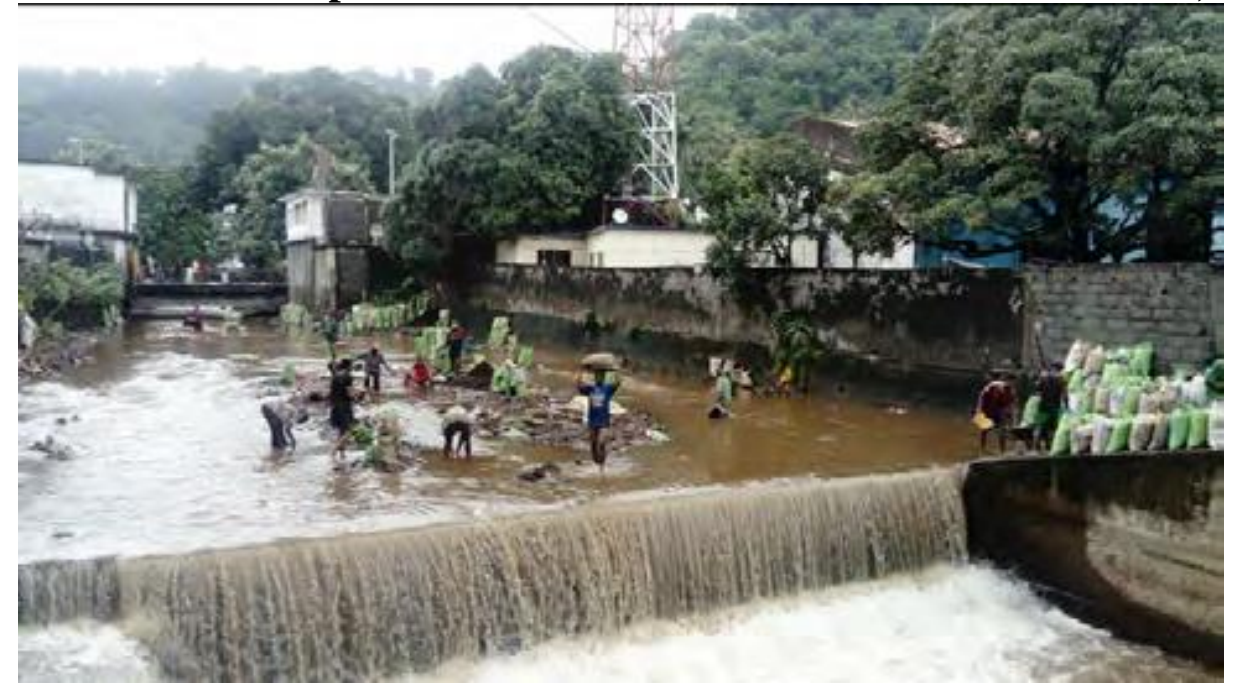


Photo $^{\circ} 7$ : Travailleuse informelle qui collecte du sable du littoral Source : Perez, 2018.

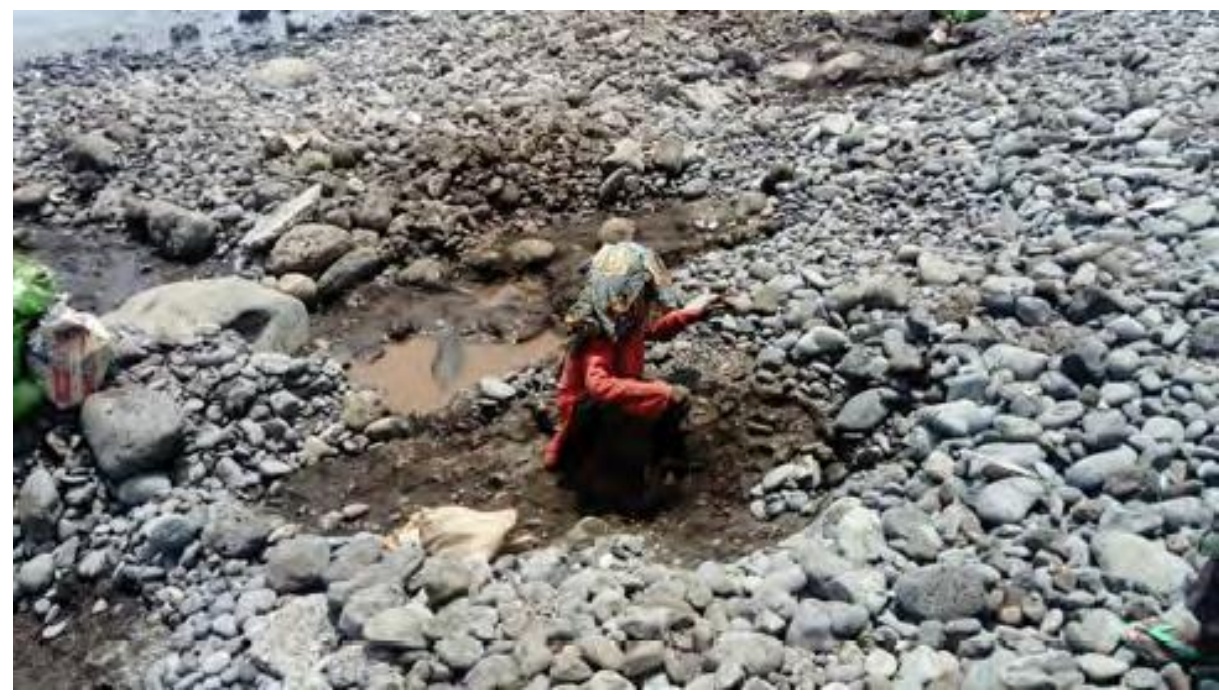

Photo ${ }^{\circ} 8$ : Effondrement d'une route de Mutsamudu Bahers, 2018.

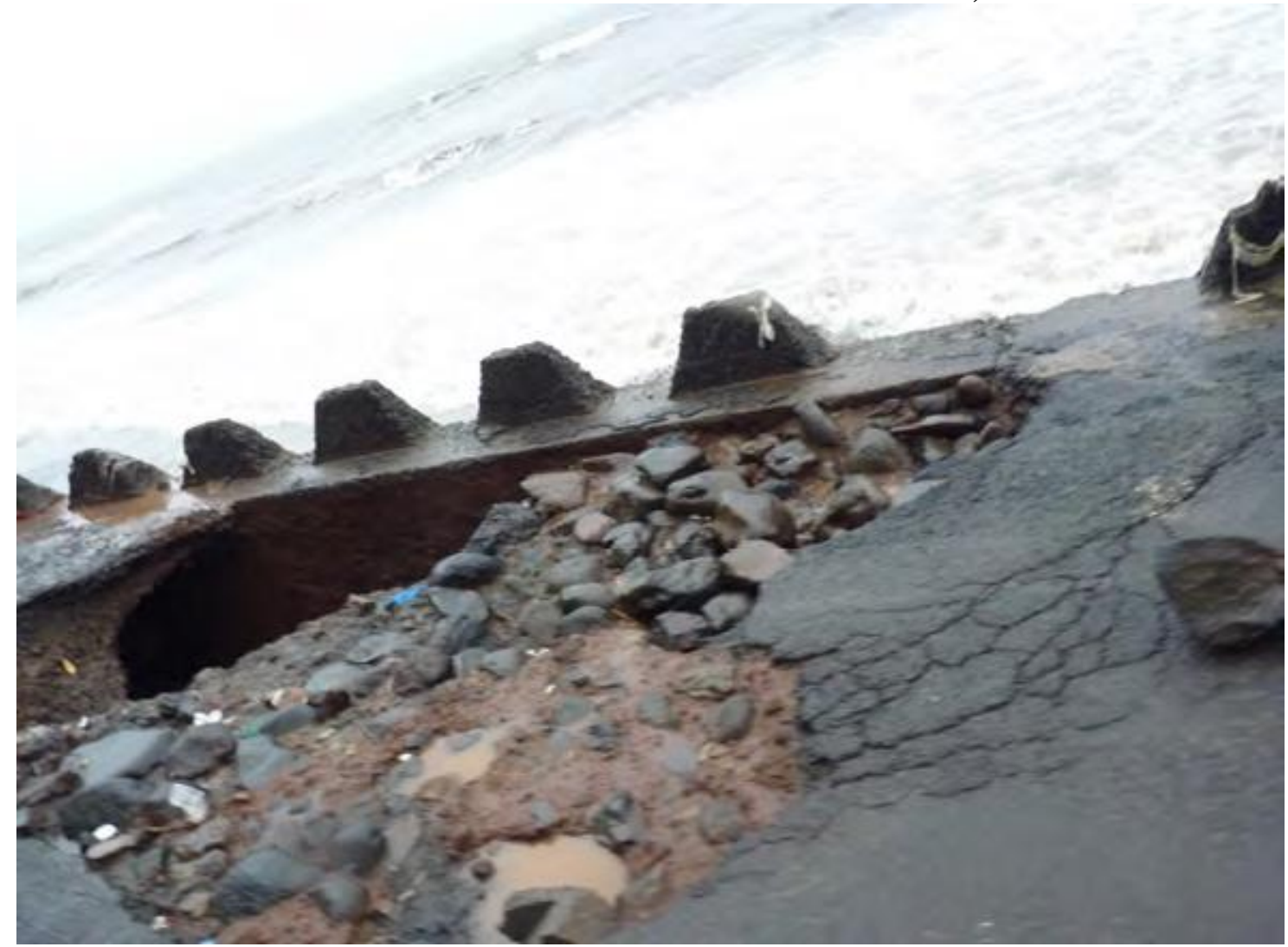

49Une dernière situation de vulnérabilité métabolique concerne le lien entre une ressource d'exploitation (le bois) et l'injonction économique à exporter des huiles essentielles d'YlangYlang. En effet, la filière de distillation d'Ylang-Ylang est un produit phare de l'économie de l'île et emploie $15 \%$ de la population active (entretien avec une chargée de mission chez Initiative Développement). Elle représente à Ndzuwani environ 270 distillations par an pour 
une production de 3 litres d'huile par distillation. Cependant, ce processus est grandement consommateur d'eau de rivière, et les assèche in fine, ainsi que de bois, ce qui est une des causes majeures de la déforestation à Ndzuwani. Il faut environ une tonne de bois par alambic et : « le plus souvent ils mandatent un bûcheron qui va repérer l'arbre et le couper. Mais aucune replantation d'arbre est effectuée après les coupes » (Entretien avec une chargée de mission Initiative Développement, 2018). Les surfaces de forêts dégradées seraient presque deux fois supérieures en 2014 par rapport à 1995 (Boussougou, Brou, Mohamed, 2015) et la production en 2016 atteint le niveau élevé de $260 \mathrm{~kg} / \mathrm{hab} / \mathrm{an}$. Ce manque de ressources propres affaiblit la résilience économique du territoire. Ainsi, cette filière de distillation d'YlangYlang articule des circuits de différents flux qui produisent des vulnérabilités territoriales (mise en difficulté de l'agroforesterie insulaire), matérielles (forte consommation de bois et d'eau et fragilités environnementales) et sociales (dépendance d'un secteur d'emploi à ces exportations). Par ailleurs, même sur ce marché de niche, la concurrence est élevée à l'échelle mondiale, ce qui implique une course à la production et aux rendements peu en adéquation avec les capacités du territoire. L'analyse des rapports de forces entre l'île et les territoires d'exportation est au cœur de cette vulnérabilité métabolique, car elle met en exergue la tension entre le besoin économique d'exportations de marchandises, la demande en forte hausse de pays importateurs (notamment des entreprises chinoises) et la nécessité d'engager beaucoup de ressources insulaires.

\section{Les atouts d'un métabolisme insulaire et émergent : entre sobriété et informalité}

50Malgré ces situations de vulnérabilités métaboliques, le métabolisme de l'île de Ndzuwani présente de nombreux signaux de transition. Tout d'abord, de nombreux acteurs, issus des ONG locales et internationales, mettent en œuvre des solutions in situ issues des pratiques vernaculaires. Il s'agit par exemple de sacs recyclés à base de papier et tissus récupérés, de fours qui optimisent la combustion du bois, de tapis réalisés à partir de restes de sandales plastiques (très nombreuses dans les dépôts sauvages de déchets sur les littoraux), de marmites faites de canettes d'aluminium, de briques de cartons pour les cheminées, de nappes réalisées par des groupes de femmes à partir de textiles usagés. Ces solutions, certes modestes en termes de volumes, attestent d'un dynamisme local et de capacités collectives.

$\underline{51 P a r}$ ailleurs, de nombreux auteurs citent la sobriété matérielle comme une perspective de durabilité pour les territoires (Bourg, Papaux, 2010). À Ndzuwani, on peut vraisemblablement considérer qu'il s'agit déjà d'une société sobre (même s'il faut noter que l'accès aux ressources n'est pas assuré de manière complètement homogène dans la population). Si la consommation matérielle (DMC) en France atteint environ 12 t/hab/an (13,5 t/hab/an dans l'Union européenne), ce niveau de consommation [7][7]Sans prendre en compte les flux indirects associés aux... est neuf fois supérieur à celui de Ndzuwani, dont la consommation matérielle est de 1,4 t/hab/an. Cet indicateur reste peu élevé par rapport à d'autres territoires insulaires émergents : la DMC aux Philippines est d'environ 6 t/hab/an (Martinico-Perez et alii, 2018) et à Trinidad d'environ 17 t/hab/an (Krausmann, Richter, Eisenmenger, 2014). Ces deux derniers pays symbolisent des modèles économiques extractivistes, dont l'industrie est fortement orientée vers l'export de ressources locales (le minerai métallique aux Philippines et le pétrole à Trinidad), ce qui a pour conséquence des forts taux d'utilisation énergétique et de déchets issus des productions. De même, les importations de marchandises à Ndzuwani (0,4 t/hab/an) sont bien moindres par rapport aux 5 t/hab/an en France ou aux 
$8 \mathrm{t} / \mathrm{hab} / \mathrm{an}$ en Allemagne. En cela, il n'est pas vraiment essentiel de proposer des perspectives pour consommer moins aux autorités anjouanaises, mais bien d'adapter le modèle de développement de l'île pour diminuer les fragilités environnementales et sociales. A l'opposé, ce cas du Sud global, comme d'autres (Millington and Lawhon, 2018), fournit des enseignements ayant une portée mondiale et démontre l'utilité de la mobilité des idées dans l'espace (et pas seulement du Nord au Sud, mais réciproquement).

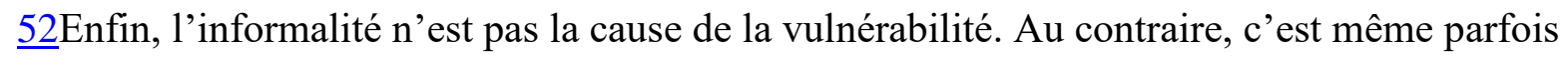
le secteur informel qui peut réduire, sur certains aspects, la vulnérabilité métabolique. La présence d'acteurs informels peut s'avérer être l'un des principaux leviers pour optimiser la gestion des flux sur l'île de Ndzuwani. D'après les entretiens, très peu de déchets résultent de l'activité de construction, contrairement aux pays du Nord global, car ils sont tous systématiquement réutilisés sur les chantiers, par manque de matières premières accessibles : «La filière de construction est très informelle, la population ne pouvant pas financer des matériaux neufs réutilise les déchets de construction et réalise même des cotisations communautaires afin de construire les routes et mosquées de leur quartier ou village » (Entretien d'un habitant construisant sa maison, 2018). Ces matières secondaires sont donc pleinement réintégrées localement aux cycles de production et donnent lieu à différents registres d'organisation collective. L'enjeu, une fois les flux bien identifiés et séparés, est de trouver le modèle organisationnel permettant d'intégrer les contraintes des différents acteurs et d'offrir un mode de gestion des territoires adapté aux moyens localement disponibles.

Ainsi, cette catégorie de l'informalité, variée et offrant d'autres moyens d'analyse que le seul angle socioprofessionnel, apporte plusieurs pistes de lecture métabolique : le secteur informel est à la fois un producteur de flux, un opérateur de pratiques innovantes et engagées, comme le montrent d'autres recherches (McFarlane, 2012), et probablement un rempart à une vulnérabilité accrue du territoire. Sans nier les incommodités du travail des récupérateurs informels de sable, de bois ou de déchets, l'analyse du métabolisme montre son rôle prépondérant dans l'économie des flux.

\section{Conclusion : l'approche empirique des flux pour comprendre les vulnérabilités métaboliques}

53Notre enquête met en évidence la nécessité d'ouvrir les études du métabolisme territorial aux dimensions sociales, politiques et organisationnelles pour comprendre les défis et atouts de la circulation des flux de matière et d'énergie en milieu insulaire. L'observation de ces métabolismes implique ainsi de s'attacher à identifier leurs modalités de gouvernance (Garcier, Rocher, Verdeil, 2017), les conflictualités qui en résultent (Demaria, Schindler, 2016) et les politiques qui les instruisent (Cousins, Newell, 2015). Un des premiers freins dans cette recherche fut de produire une compilation de données utiles tant sur le plan de la comptabilisation que sur la connaissance des pratiques d'acteurs, indispensables à une analyse « «étendue » (Pincetl, Bunje, Holmes, 2012 ; Pincetl, Newell, 2017) et « située » (Lawhon, Ernston, Silver, 2014 ; Martinez-Alier, Temper, Demaria, 2016) du métabolisme territorial. Nous avons montré la nécessité méthodologique d'une approche empirique des flux pour comprendre les vulnérabilités métaboliques. La première piste que nous avons explorée concerne la mobilisation de l'expertise par les acteurs locaux, en tant que stratégie de contournement pour l'estimation des flux non couverts par la statistique. La seconde piste a 
permis de quantifier, par l'observation, la matérialité de certains flux et les « situations métaboliques ». Ainsi, quelques matières sont caractéristiques des situations de vulnérabilité : la biomasse alimentaire, le sable et le bois.

$\underline{54 U n e ~ a u t r e ~ p e r s p e c t i v e ~ c o n c e r n e ~ l e s ~ f o r c e s ~ d u ~ m e ́ t a b o l i s m e ~ i n s u l a i r e ~ d e ~ N d z u w a n i . ~ M a l g r e ́ ~}$ les difficultés rencontrées par les acteurs, l'informalité pourrait être une source de solution vers un métabolisme plus résilient. Il s'agirait de développer des modèles organisationnels et territoriaux ad hoc pour une meilleure gestion des ressources et des déchets. Enfin, si le territoire manque terriblement d'infrastructures et de ressources propres, il n'en reste pas moins que des solutions sont mises en œuvre et que la sobriété matérielle est réelle.

55Enfin, cet article ouvre des perspectives sur le cadre théorique des vulnérabilités métaboliques, qui prolongent le concept de vulnérabilité environnementale. Si les travaux se référant à la vulnérabilité environnementale (Mathis et alii, 2016) s'attachent à interroger les dispositions d'une société à être plus ou moins bien préparée à supporter un changement brutal de son environnement, la vulnérabilité métabolique s'intéresse au prolongement de ces modifications perturbatrices dans l'analyse de la circulation des ressources, au travers de leur extraction, de leur production et de leur fin de vie en tant que déchets, entre territoires de consommation et territoires d'approvisionnement et de rejets. Des données supplémentaires sur les autres «chaînes » de ressources et les trajectoires socio-écologiques du territoire pourraient participer à consolider cette perspective. En cela, ce concept contribue à une meilleure connaissance de l'écologie territoriale (Barles, 2017 ; Buclet, 2015) de ces milieux insulaires, mais pas seulement. En effet, l'intérêt heuristique de cette approche métabolique socio-matérielle ne se limite pas aux espaces strictement insulaires, qui sont in fine très dépendants du poids des circulations logistiques et des déterminations géopolitiques. L'insularité révèle, voire exacerbe, des contraintes métaboliques que rencontrent également d'autres territoires physiquement enclavés, isolés par les axes de transport ou séparés par des frontières politiques. Leurs relations avec les territoires d'approvisionnements et de rejets et leur volonté de plus d'autonomie et d'autosuffisance sont des déterminants comparables aux territoires insulaires. Les leçons tirées de ces cas d'études permettent donc non seulement d'être appliquées à d'autres territoires, mais aussi d'identifier des approches méthodologiques pour comprendre la façon dont circulent les flux, et les types de relations sociales et politiques qui les régissent ou qu'ils induisent.

\section{Notes}

- $[1]$

Traduit de MFA : Material Flow Analysis.

- $[2]$

Que nous traduisons par « métabolisme énergétique insulaire ».

- $[3]$

Ilhadas Flores, de Jorge Furtado, Brésil, 1989.

- $[4]$ 
L'île Henderson est une des quatre îles Pitcairn, territoire britannique d'outre-mer.

- $[5]$

Expression empruntée à Romain Garcier et Laurence Rocher lors du séminaire de l'atelier 2 de l'UMR EVS « La Nouvelle-Calédonie face à ses déchets » (14/05/2019).

- [6]

Travail de recherche mené dans le cadre d'un partenariat entre le laboratoire UMR ESO-Le Mans et le Gouvernorat de Ndzuwani. Jean-Baptiste Bahers et Mathieu Durand ont effectué une mission courte en début d'enquête (10 jours), quand Jeanne Perez a collecté le détail des données sur une plus longue période (2 mois).

- $\quad[7]$

Sans prendre en compte les flux indirects associés aux importations, qui sont bien plus importants en Europe.

Bibliographie

Allen M.G., 2017, Islands, extraction and violence: Mining and the politics of scale in Island Melanesia, Political Geography 57, p. 81-90. DOI: 10.1016/j.polgeo.2016.12.004

Bahers J.-B., 2011, Écologie industrielle, gestion des déchets et territoire insulaire. L'exemple de I'archipel des Fidji, in: Taglioni F. (sous la direction de), Insularité et développement durable, Marseille : IRD Éditions, p. 162-173.

Bahers J.-B., Barles S., Durand M., 2019, Urban Metabolism of Intermediate Cities: The Material Flow Analysis, Hinterlands and the Logistics-Hub Function of Rennes and Le Mans (France), Journal of Industrial Ecology, 23-3, p. 686-698. DOI: 10.1111/jiec.12778

Bahers J.-B., Durand M., Beraud H., 2017, Quelle territorialité pour l'économie circulaire ? Interprétation des typologies de proximité dans la gestion des déchets, Flux, 2017/3-4 ( $\left.N^{\circ} 109-110\right)$, p. 129-141. DOI: 10.3917/flux1.109.0129

Bahers J.-B., Giacchè G., 2019, Towards a metabolic rift analysis: The case of urban agriculture and organic waste management in Rennes (France), Geoforum, 98, p. 97-107. DOI:

10.1016/j.geoforum.2018.10.017

Barles S., 2010, Society, energy and materials: the contribution of urban metabolism studies to sustainable urban development issues, Journal of Environmental Planning and Management, vol. 53, p. $439-455$. 
Barles S., 2017, Écologie territoriale et métabolisme urbain : quelques enjeux de la transition socioécologique, Revue d'Économie Régionale \& Urbaine Décembre, p. 819-836. DOI:

10.3917/reru.175.0819

Baynes T.M., Wiedmann T., 2012, General approaches for assessing urban environmental sustainability, Current Opinion in Environmental Sustainability, Human settlements and industrial systems, vol 4, p. 458-464. DOI: 10.1016/j.cosust. 2012.09.003

Belghith N. B. H., Takamatsu S., Razafimanantsoa Harivelo F.N., Sharma N., Mahdi S., 2018. Comoros Poverty Assessment (No. 125069), Washington: The World Bank.

Blanchy S., 2015, Anjouan (Comores), un nœud dans les réseaux de l'océan Indien. Émergence et rôle d'une société urbaine lettrée et marchande (XVIle-XXe siècle), Afriques. Débats, méthodes et terrains d'histoire. DOI: 10.4000/afriques.1817

Bocquet D., 2013, Singapour ville durable ? Innovations et limites d'une politique environnementale et urbaine, Petits déjeuners de la chaire ville de l'École des Ponts. Série Green Cities.

Bond A., Lavers J., 2017, Pristine paradise to rubbish dump: the same Pacific island, 23 years apart [WWW Document], The Conversation. [Online] Available at: URL http://theconversation.com/pristine-paradise-to-rubbish-dump-the-same-pacific-island-23-yearsapart-80811 (accessed 11.26.18).

Bouchard C., Marrou L., Plante S., Payet R., Duchemin E., 2011, Les petits États et territoires insulaires face aux changements climatiques : vulnérabilité, adaptation et développement, VertigO la revue électronique en sciences de l'environnement. DOI: 10.4000/vertigo.10634

Bourg D., Papaux A., 2010, Vers une société sobre et durable, 1ère éd., Paris : Presses Universitaires de France-PUF.

Boussougou G. B., Brou Y. T., Mohamed I., 2015, Changements de la couverture forestière dans I'île d'Anjouan entre 1995 et 2014, Actes de la 11ème conférence internationale annuelle Spatial Analysis and GEOmatics - SAGEO, Hammamet, Tunisia, November 23th.

Breetz H.L., 2017, Political-industrial ecology: Integrative, complementary, and critical approaches. Geoforum, vol. 85, p. 392-395. DOI: 10.1016/j.geoforum.2016.11.011

Buclet N.(sous la direction de), 2015, Essai d'écologie territoriale. L'exemple d'Aussois en Savoie, Paris : CNRS Éditions.

Cecchin A., 2016, Material flow analysis for a sustainable resource management in island ecosystems, Journal of Environmental Planning and Management, vol. 60(9), p. 1640-1659. DOI:

10.1080/09640568.2016.1246997

Chrysoulakis N., Lopes M., San José R., Grimmond C. S. B., Jones M. B., Magliulo V., Klostermann J. E. M., Synnefa A., Mitraka Z., Castro E. A., González A., Vogt R., Vesala T., Spano D., Pigeon G., FreerSmith P., Staszewski T., Hodges N., Mills G., Cartalis C., 2013, Sustainable urban metabolism as a link 
between bio-physical sciences and urban planning: The BRIDGE project, Landscape and Urban Planning, Vol. 112, p. 100-117. DOI: 10.1016/j.landurbplan.2012.12.005

Cole S., 2012. A political ecology of water equity and tourism: A Case Study From Bali, Annals of Tourism Research, 39, p. 1221-1241. DOI: 10.1016/j.annals.2012.01.003

Cousins J. J., Newell J. P., 201, A political-industrial ecology of water supply infrastructure for Los Angeles, Geoforum, 58, p. 38-50. DOI: 10.1016/j.geoforum.2014.10.011

Demaria F., Schindler S., 2016, Contesting Urban Metabolism: Struggles Over Waste-to-Energy in Delhi, India, Antipode, 48, p. 293-313. DOI: 10.1111/anti.12191

D’Ercole R., Metzger P., 2009, Las dimensiones espaciales del manejo de crisis. Interés de la investigación y aplicación en Quito, Bulletin de l'Institut français d'études andines, p. 893-915. DOI: 10.4000/bifea.2543

Deschenes P. J., Chertow M., 2004, An island approach to industrial ecology: towards sustainability in the island context, Journal of Environmental Planning and Management, 47, p. 201-217. DOI: 10.1080/0964056042000209102

Durand M., 2018, Quand la vulnérabilité des populations permet de réduire la vulnérabilité urbaine : les déchets à Lima (Pérou), in: Cirelli C., Florin B. (Sous la direction de), Sociétés Urbaines et Déchets : Éclairages Internationaux, Perspectives Villes et Territoires, Tours : Presses universitaires FrançoisRabelais, p. 317-345.

Durand M., De Oliveira Neves F., 2019, L'intégration des cueilleurs de déchets latino-américains ou la création d'une nouvelle marge, EchoGéo, vol. 47. DOI: 10.4000/echogeo.16894

Durand M., Metzger P., 2009, Gestión de residuos y transferencia de vulnerabilidad en Lima/Callao, Bulletin de I'Institut français d'études andines, vol. 38 (3), p. 623-646. DOI: 10.4000/bifea.2396

Durand M., Popescu R., D'Ercole R., 2015, Les déchets post-catastrophe à Haïti : les jeux d'acteurs d'une gestion informelle, VertigO - la revue électronique en sciences de l'environnement, vol. 15 (1). DOI: $10.4000 /$ vertigo.15970

Duro J. A., Schaffartzik A., Krausmann F., 2018, Metabolic Inequality and Its Impact on Efficient Contraction and Convergence of International Material Resource Use, Ecological Economics 145, p. 430-440. DOI: 10.1016/j.ecolecon.2017.11.029

Eckelman M. J., Chertow M. R., 2009, Using Material Flow Analysis to Illuminate Long-Term Waste Management Solutions in Oahu, Hawaii, Journal of Industrial Ecology, 13, p. 758-774. DOI: 10.1111/j.1530-9290.2009.00159.x

Eurostat, 2009, Economy wide material flow accounts: Compilation guidelines for reporting to the 2009 Eurostat questionnaire, Luxembourg: Office for Official Publications of the European Communities.

FAOSTAT (the Food and Agriculture Organization Statistics Division), 2018, FAO statistical database, Rome: Food and Agriculture Organization. [Online] Available at: http://www.fao.org/faostat/fr/\#country/45 (consulté le 30/11/2018). 
Fetzel T., Petridis P., Noll D., Singh S. J., Fischer-Kowalski M., 2018, Reaching a socio-ecological tipping point: Overgrazing on the Greek island of Samothraki and the role of European agricultural policies, Land Use Policy, 76, p. 21-28. DOI: 10.1016/j.landusepol.2018.04.042

Fischer-Kowalski M., Krausmann F., Giljum S., Lutter S., Mayer A., Bringezu S., Moriguchi Y., Schütz H., Schandl H., Weisz H., 2011, Methodology and Indicators of Economy-wide Material Flow Accounting, Journal of Industrial Ecology, vol. 15, p. 855-876. DOI: 10.1111/j.1530-9290.2011.00366.x

Fouquet E., 2014, La gestion des déchets ménagers dans la baie de Mutsamudu (île d’Anjouan Union des Comores), Mémoire de recherche, Université du Mans, Master 1 Ville et Environnements Urbains.

Garcier R., Martinais E., Rocher L., 2017, Désigner, mesurer, réguler : la mise en politique des flux et circulations, Géocarrefour, vol. 91 (3). [En ligne] (consulté le 21 aout 2019) Disponible à l'adresse : http://journals.openedition.org.inshs.bib.cnrs.fr/geocarrefour/10362

Garcier R., Rocher L., Verdeil É., 2017, Introduction : circulation des matières, économies de la circularité, Flux, 2017/2 (N¹08), p. 1-7. DOI: 10.3917/flux1.108.0001

Gérard Y., 2009, Étalement urbain et transformation de la structure urbaine de deux capitales insulaires : Moroni et Mutsamudu, archipel des Comores (océan Indien), Les Cahiers d'Outre-Mer. Revue de géographie de Bordeaux, vol. 62, p. 513-528. DOI: 10.4000/com.5781

Ginard-Bosch F. J., Ramos-Martín J., 2016, Energy metabolism of the Balearic Islands (1986-2012), Ecological Economics, vol. 124, p.25-35. DOI: 10.1016/j.ecolecon.2015.12.012

Gössling S., 2003, Tourism and Development in Tropical Islands: Political Ecology Perspectives, Cheltenham : Edward Elgar.

Guibrunet L., Sanzana Calvet M., Castán Broto V., 2016, Flows, system boundaries and the politics of urban metabolism: Waste management in Mexico City and Santiago de Chile, Geoforum, vol. 85. DOI: 10.1016/j.geoforum.2016.10.011

Harrison C., Popke J., 2018, Geographies of renewable energy transition in the Caribbean: Reshaping the island energy metabolism, Energy Research \& Social Science, vol. 36, p. 165-174. DOI:

10.1016/j.erss.2017.11.008

Hird M. J., 2017, Waste, Environmental Politics and Dis/Engaged Publics, Theory, Culture \& Society, vol. 34, 187-209. DOI: 10.1177/0263276414565717

Kakeu-Tardy R. C. M., 2018, Secteur informel-formel et espace urbain à Bafoussam (Cameroun) : la récupération des déchets solides municipaux, L'espace géographique, vol. 47, p. 261-281.

Krausmann F., Gingrich S., Eisenmenger N., Erb K.-H., Haberl H., Fischer-Kowalski M., 2009, Growth in global materials use, GDP and population during the 20th century, Ecological Economics, vol. 68, p. 2696-2705. DOI: 10.1016/j.ecolecon.2009.05.007

Krausmann F., Lauk C., Haas W., Wiedenhofer D., 2018, From resource extraction to outflows of wastes and emissions: The socioeconomic metabolism of the global economy, 1900-2015, Global Environmental Change, vol. 52, p. 131-140. DOI: 10.1016/j.gloenvcha.2018.07.003 
Krausmann F., Richter R., Eisenmenger N., 2014, Resource Use in Small Island States, Journal of Industrial Ecology, vol.18, p. 294-305. DOI: 10.1111/jiec.12100

Lawhon M., Ernstson H., Silver J., 2014, Provincializing Urban Political Ecology: Towards a Situated UPE Through African Urbanism, Antipode, vol.46, p. 497-516. DOI: 10.1111/anti.12051

Legoff N., 2011, Les Comores et l'aléa cyclonique dans le contexte des changements climatiques : la vulnérabilité différenciée d'Anjouan et de Mayotte, VertigO - la revue électronique en sciences de I'environnement, vol. 10(3). DOI: 10.4000/vertigo.10497

Martinez-Alier J., Temper L., Demaria F., 2016, Social Metabolism and Environmental Conflicts in India, in: Ghosh N., Mukhopadhyay P., Shah A., Panda M. (eds), Nature, Economy and Society: Understanding the Linkages, New Delhi: Springer India, p. 19-49.

Martinico-Perez M. F. G., Schandl H., Fishman T., Tanikawa H., 2018, The Socio-Economic Metabolism of an Emerging Economy: Monitoring Progress of Decoupling of Economic Growth and Environmental Pressures in the Philippines, Ecological Economics, vol. 147, p. 155-166. DOI:

10.1016/j.ecolecon.2018.01.012

Marty P., 2013, Les appropriations urbaines de la question agricole. Le cas de Brive, de 1945 à 2012 Thèse de doctorat en aménagement, sous la direction de Sabine Barles, Université Paris 1.

Mathieu N., 2006, Pour une construction interdisciplinaire du concept de milieu urbain durable, Call for building the interdisciplinary concept of sustainable urban milieu, Natures Sciences Sociétés, 14, p. 376-382.

Mathis C.-F., Frioux S., Dagenais M., Walter F., 2016, Vulnérabilités environnementales : perspectives historiques, VertigO - la revue électronique en sciences de l'environnement, vol 16(3). DOI: 10.4000/vertigo.17993

McFarlane C., 2012, Rethinking Informality: Politics, Crisis, and the City, Planning Theory \& Practice, vol.13, p. 89-108. DOI: 10.1080/14649357.2012.649951

McFarlane C., 2013, Metabolic inequalities in Mumbai, City, vol. 17, p. 498-503.

Millington N., Lawhon M., 2018, Geographies of waste: Conceptual vectors from the Global South, Progress in Human Geography, (En ligne avant publication). DOI: 10.1177/0309132518799911

Monsaingeon B., 2017, Homo detritus - Critique de la société du déchet, Paris : Le Seuil.

Newell J. P., Cousins J. J., 2015, The boundaries of urban metabolism: Towards a political-industrial ecology, Progress in Human Geography, vol. 39, p. 702-728. DOI: 10.1177/0309132514558442

Oppio A., Corsi S., 2017, Territorial vulnerability and local conflicts perspectives for waste disposals siting. A case study in Lombardy region (Italy), Journal of Cleaner Production, vol. 141, p. 1528-1538. DOI: 10.1016/j.jclepro.2016.09.203

Pelling M., Uitto J. I., 2001, Small island developing states: natural disaster vulnerability and global change, Global Environmental Change Part B: Environmental Hazards, vol. 3, p.49-62. DOI:

10.1016/S1464-2867(01)00018-3 
Petridis P., Fischer-Kowalski M., 2016, Island Sustainability: The Case of Samothraki, in: Haberl H., Fischer-Kowalski M., Krausmann F., Winiwarter V. (Eds.), Social Ecology, Cham: Springer International Publishing, p. 543-557.

Perez J., 2018, Quel potentiel la connaissance du métabolisme territorial de l'île d'Anjouan offre-t-il en termes de mise en œuvre de l'économie circulaire ?, Mémoire de recherche, Université du Mans, Master 1 Ville et Environnements Urbains.

Pincetl S., Bunje P., Holmes T., 2012, An expanded urban metabolism method: Toward a systems approach for assessing urban energy processes and causes, Landscape and Urban Planning, vol. 107, p. 193-202. DOI: 10.1016/j.landurbplan.2012.06.006

Pincetl S., Newell J. P., 2017, Why data for a political-industrial ecology of cities?, Geoforum, vol 85. DOI: 10.1016/j.geoforum.2017.03.002

Robineau C., 1966, Société et économie d'Anjouan (Océan Indien), Mémoires ORSTOM. Paris : ORSTOM.

SchandI H., Fischer-Kowalski M., West J., Giljum S., Dittrich M., Eisenmenger N., Geschke A., Lieber M., Wieland H., Schaffartzik A., Krausmann F., Gierlinger S., Hosking K., Lenzen M., Tanikawa H., Miatto A., Fishman T., 2018, Global Material Flows and Resource Productivity: Forty Years of Evidence, Journal of Industrial Ecology, 22, p. 827-838. DOI: 10.1111/jiec.12626

Sinane K., David G., Pennober G., Troadec R., 2011, Fragilisation et modification des formations littorales meubles sur l'île d'Anjouan (Comores) : Quand l'érosion d'origine anthropique se conjugue au changement climatique, VertigO - la revue électronique en sciences de l'environnement, vol 10(3). DOI: 10.4000/vertigo.10528

Singh S. J., Grünbühel C. M., Schandl H., Schulz N., 2001, Social Metabolism and Labour in a Local Context: Changing Environmental Relations on Trinket Island, Population and Environment 23, p. 71104. DOI: 10.1023/A:1017564309651

Singh S. J., Haberl H., Chertow M., Mirtl M., Schmid M. (Eds.), 2013, Long Term Socio-Ecological Research: Studies in Society-Nature Interactions Across Spatial and Temporal Scales, HumanEnvironment Interactions, Dordrecht: Springer Netherlands.

Taglioni F., 2008, L'île d'Anjouan, figure de la balkanisation de l'archipel des Comores, EchoGéo, vol. «Sur le vif 2008 ». DOI: 10.4000/echogeo.7223

Thompson B. S., 2018, The political ecology of mangrove forest restoration in Thailand: Institutional arrangements and power dynamics, Land Use Policy, 78, p. 503-514. DOI:

10.1016/j.landusepol.2018.07.016

Tongnuanchan P., Benjakul S., 2014, Essential Oils: Extraction, Bioactivities, and Their Uses for Food Preservation, Journal of Food Science, vol. 79, R1231-R1249. DOI: 10.1111/1750-3841.12492

USGS (U.S. Geological Survey), 2010, Minerals information. Reston, VA, USA: U.S. Geological Survey, [Online] Available at : http://minerals.usgs.gov/minerals/ (Consulté le 30/11/2018). 
Wachsmuth D., 2012, Three Ecologies: Urban Metabolism and the Society-Nature Opposition, The Sociological Quarterly, vol. 53, p. 506-523. DOI: 10.1111/j.1533-8525.2012.01247.x

Wiedenhofer D., Fishman T., Lauk C., Haas W., Krausmann F., 2019, Integrating Material Stock Dynamics Into Economy-Wide Material Flow Accounting: Concepts, Modelling, and Global Application for 1900-2050, Ecological Economics, vol. 156, p. 121-133. DOI:

10.1016/j.ecolecon.2018.09.010

Wœssner R., 2016, Dubaï et Abou Dhabi : la naissance d'un emporium, Revue d'Économie Régionale et Urbaine, 2016/1 (Février), p. 155-174. DOI : 10.3917/reru.161.0155

Wu-Tiu-Yen N., 2015, L'immigration clandestine à Mayotte : un phénomène révélateur de l'incidence des changements climatiques sur la sécurité humaine? Note de recherche, VertigO - la revue électronique en sciences de l'environnement, Hors-série 21. DOI: 10.4000/vertigo.15798 